\title{
First low-latency LIGO+Virgo search for binary inspirals and their electromagnetic counterparts
} B. Allen ${ }^{9,14,10}$, E. Amador Ceron ${ }^{14}$, D. Amariutei ${ }^{15}$, S. B. Anderson ${ }^{1}$, W. G. Anderson ${ }^{14}$, K. Arai $^{1}$, M. A. Arain ${ }^{15}$, M. C. Araya ${ }^{1}$, S. M. Aston ${ }^{16}$, P. Astone ${ }^{17}$, D. Atkinson ${ }^{19}$ P. Aufmuth ${ }^{10,9}$, C. Aulbert ${ }^{9,10}$, B. E. Aylott ${ }^{16}$, S. Babak ${ }^{20}$, P. Baker ${ }^{21}$, G. Ballardin ${ }^{22}$, S. Ballmer ${ }^{23}$, J. C. B. Barayoga ${ }^{1}$, D. Barker ${ }^{19}$, F. Barone D $^{5,7}$, B. Barr ${ }^{3}$, L. Barsotti2 ${ }^{24}$, M. Barsuglia ${ }^{25}$, M. A. Barton ${ }^{19}$, I. Bartos ${ }^{26}$, R. Bassiri ${ }^{3}$, M. Bastarrika ${ }^{3}$, A. Basti ${ }^{27,28}$, J. Batch ${ }^{19}$, J. Bauchrowitz, ${ }^{9}$, Th. S. Bauer ${ }^{11}$, M. Bebronne ${ }^{4}$, D. Beck ${ }^{30}$, B. Behnke ${ }^{20}$, M. Bejger ${ }^{33}$, M.G. Beker ${ }^{11}$, A. S. Bell ${ }^{3}$, A. Belletoile ${ }^{4}$, I. Belopolski ${ }^{26}$, M. Benacquista ${ }^{37}$, J. M. Berliner ${ }^{19}$, A. Bertolini ${ }^{9,10}$, J. Betzwieser ${ }^{1}$, N. Beveridge ${ }^{3}$, P. T. Beyersdorf ${ }^{38}$, I. A. Bilenko ${ }^{39}$, G. Billingsley ${ }^{1}$, J. Birch $^{8}$, R. Biswas ${ }^{37}$, M. Bitossi ${ }^{27}$, M. A. Bizouard ${ }^{40}$, E. Black ${ }^{1}$, J. K. Blackburn ${ }^{1}$, L. Blackburn ${ }^{42}$, D. Blair ${ }^{43}$, B. Bland ${ }^{19}$, M. Blom ${ }^{11}$, O. Bock ${ }^{9,10}$, T. P. Bodiya ${ }^{24}$, C. Bogan ${ }^{9,10}$ R. Bondarescu ${ }^{44}$, F. Bondu ${ }^{46}$, L. Bonelli ${ }^{27,28}$, R. Bonnand ${ }^{47}$, R. Bork ${ }^{1}$, M. Born ${ }^{9,10}$, V. Boschi ${ }^{27}$, S. Bose ${ }^{48}$, L. Bosi ${ }^{49}$, B. Bouhou ${ }^{25}$, S. Braccini ${ }^{27}$, C. Bradaschia ${ }^{27}$, P. R. Brady ${ }^{14}$ V. B. Braginsky ${ }^{39}$, M. Branchesi $5^{51,52}$, J. E. Brau ${ }^{53}$, J. Breyer ${ }^{9,10}$, T. Briant ${ }^{54}$, D. O. Bridges ${ }^{8}$, A. Brillet ${ }^{45}$, M. Brinkmann ${ }^{9,10}$, V. Brisson ${ }^{40}$, M. Britzger ${ }^{9,10}$, A. F. Brooks ${ }^{1}$, D. A. Brown ${ }^{23}$, T. Bulik ${ }^{32}$, H. J. Bulten ${ }^{11,12}$, A. Buonanno ${ }^{55}$, J. Burguet-Castel1 ${ }^{56}$, D. Buskulic ${ }^{4}$, C. Buy ${ }^{25}$, R. L. Byer ${ }^{30}$, L. Cadonati ${ }^{57}$, G. Cagnoli ${ }^{51}$, E. Calloni ${ }^{5,6}$, J. B. Camp $^{42}$, P. Campsie ${ }^{3}$, J. Cannizzo ${ }^{42}$, K. Cannon ${ }^{58}$, B. Canuel ${ }^{22}$, J. Cao ${ }^{59}$, C. D. Capano ${ }^{23}$, F. Carbognani ${ }^{22}$, L. Carbone ${ }^{16}$, S. Caride ${ }^{60}$, S. Caudill ${ }^{61}$, M. Cavaglià ${ }^{62}$, F. Cavalier ${ }^{40}$, R. Cavalieri ${ }^{22}$, G. Cella ${ }^{27}$, C. Cepeda ${ }^{1}$, E. Cesarini ${ }^{52}$, O. Chaibi ${ }^{45}$, T. Chalermsongsak ${ }^{1}$, P. Charlton ${ }^{63}$, E. Chassande-Mottin ${ }^{25}$, S. Chelkowski ${ }^{16}$, W. Chen ${ }^{59}$, X. Chen ${ }^{43}$, Y. Chen ${ }^{64}$, A. Chincarini ${ }^{65}$, A. Chiummo ${ }^{22}$, H. S. Cho ${ }^{66}$, J. Chow ${ }^{67}$, N. Christensen ${ }^{68}$, S. S. Y. Chua ${ }^{67}$, C. T. Y. Chung ${ }^{69}$, S. Chung ${ }^{43}$, G. Ciani ${ }^{15}$, F. Clara ${ }^{19}$, D. E. Clark ${ }^{30}$, J. Clark ${ }^{70}$, J. H. Clayton ${ }^{14}$, F. Cleva ${ }^{45}$, E. Coccia ${ }^{71,72}$, P.-F. Cohadon ${ }^{54}$, C. N. Colacino ${ }^{27,28}$, J. Colas ${ }^{22}$, A. Colla ${ }^{17,18}$, M. Colombini ${ }^{18}$, A. Conte ${ }^{17,18}$, R. Conte ${ }^{74}$, D. Cook ${ }^{19}$, T. R. Corbitt ${ }^{24}$, M. Cordier $^{38}$, N. Cornish $^{21}$, A. Corsi ${ }^{1}$, C. A. Costa ${ }^{61}$, M. Coughlin ${ }^{68}$, J.-P. Coulon ${ }^{45}$, P. Couvares ${ }^{23}$, D. M. Coward ${ }^{43}$, M. Cowart ${ }^{8}$, D. C. Coyne ${ }^{1}$, J. D. E. Creighton ${ }^{14}$, T. D. Creighton ${ }^{37}$, A. M. Cruise ${ }^{16}$, A. Cumming ${ }^{3}$, L. Cunningham ${ }^{3}$, E. Cuoco $^{22}$, R. M. Cutler ${ }^{16}$, K. Dahl ${ }^{1,10}$, S. L. Danilishin ${ }^{39}$, R. Dannenberg ${ }^{1}$, S. D'Antonio ${ }^{71}$, K. Danzmann ${ }^{9,10}$, V. Dattilo' ${ }^{22}$, B. Daudert ${ }^{1}$, H. Daveloza ${ }^{37}$, M. Davier ${ }^{40}$, E. J. Daw ${ }^{75}$, R. Day ${ }^{22}$, T. Dayanga ${ }^{48}$, R. De Rosa ${ }^{5,6}$, D. DeBra ${ }^{30}$, G. Debreczeni ${ }^{76}$, W. Del Pozzo ${ }^{11}$, M. del Prete ${ }^{78}$, T. Dent ${ }^{70}$, V. Dergachev ${ }^{1}$, R. DeRosa ${ }^{61}$, R. DeSalvo ${ }^{1}$, S. Dhurandhar ${ }^{81}$, L. Di Fiore ${ }^{5}$, A. Di Lieto ${ }^{27,28}$, I. Di Palma ${ }^{9,10}$, M. Di Paolo Emilio ${ }^{71,73}$, A. Di Virgilio ${ }^{27}$, M. Díaz ${ }^{37}$, A. Dietz ${ }^{4}$, F. Donovan ${ }^{24}$, K. L. Dooley ${ }^{15}$, M. Drago ${ }^{77,78}$, R. W. P. Drever ${ }^{82}$, J. C. Driggers ${ }^{1}$, Z. Du ${ }^{59}$, J.-C. Dumas ${ }^{43}$, S. Dwyer ${ }^{24}$, T. Eberle ${ }^{9,10}$, M. Edgar ${ }^{3}$, M. Edwards ${ }^{70}$, A. Effler ${ }^{61}$, P. Ehrens ${ }^{1}$, G. Endrö́czi ${ }^{76}$, R. Engel ${ }^{1}$, T. Etzel ${ }^{1}$, K. Evans ${ }^{3}$, M. Evans ${ }^{24}$, T. Evans ${ }^{8}$, M. Factourovich ${ }^{26}$, V. Fafone ${ }^{71,72}$, S. Fairhurst ${ }^{70}$, Y. Fan ${ }^{43}$, B. F. Farr ${ }^{83}$, D. Fazi ${ }^{83}$, H. Fehrmann ${ }^{9,10}$, D. Feldbaum ${ }^{15}$, F. Feroz ${ }^{84}$, I. Ferrante ${ }^{27,28}$, F. Fidecaro ${ }^{27,28}$, L. S. Finn ${ }^{44}$, I. Fiori ${ }^{22}$, R. P. Fisher ${ }^{44}$, R. Flaminio ${ }^{47}$, M. Flanigan ${ }^{19}$, S. Foley ${ }^{24}$, E. Forsi ${ }^{8}$, L. A. Forte ${ }^{5}$, N. Fotopoulos ${ }^{1}$, J.-D. Fournier ${ }^{45}$, J. Franc ${ }^{47}$, S. Frasca ${ }^{17,18}$, F. Frasconi ${ }^{27}$, M. Frede ${ }^{9,10}$ M. Frei ${ }^{85,86}$, Z. Frei $^{87}$, A. Freise ${ }^{16}$, R. Frey ${ }^{53}$, T. T. Fricke ${ }^{61}$, D. Friedrich ${ }^{9,10}$, P. Fritschel ${ }^{24}$, V. V. Frolov ${ }^{8}$, M.-K. Fujimoto $^{13}$, P. J. Fulda ${ }^{16}$, M. Fyffe $^{8}$, J. Gair $^{84}$, M. Galimberti $^{47}$, L. Gammaitoni4 ${ }^{49,50}$, J. Garcia ${ }^{19}$, F. Garufi ${ }^{5,6}$, M. E. Gáspár ${ }^{76}$, G. Gemme ${ }^{65}$, R. Geng ${ }^{59}$, E. Genin ${ }^{22}$, A. Gennai ${ }^{27}$, L. Á. Gergely ${ }^{88}$, S. Ghosh ${ }^{48}$, J. A. Giaime $^{61,8}$, S. Giampanis ${ }^{14}$, K. D. Giardina ${ }^{8}$, A. Giazotto ${ }^{27}$, S. Gil-Casanova ${ }^{56}$, C. Gill ${ }^{3}$, J. Gleason ${ }^{15}$, E. Goetz ${ }^{9,10}$, L. M. Goggin ${ }^{14}$, G. González ${ }^{61}$, M. L. Gorodetsky ${ }^{39}$, S. Goßler ${ }^{9,10}$, R. Gouaty ${ }^{4}$, C. Graef ${ }^{9}, 10$, P. B. Graff ${ }^{84}$, M. Granata ${ }^{25}$, A. Grant ${ }^{3}$, S. Gras ${ }^{43}$, C. Gray ${ }^{19}$, N. Gray ${ }^{3}$, R. J. S. Greenhalgh ${ }^{89}$, A. M. Gretarsson ${ }^{90}$, C. Greverie ${ }^{45}$, R. Grosso ${ }^{37}$, H. Grote $^{9,10}$, S. Grunewald $^{20}$

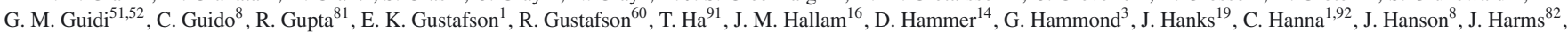
G. M. Harry ${ }^{24}$, I. W. Harry ${ }^{70}$, E. D. Harstad ${ }^{53}$, M. T. Hartman ${ }^{15}$, K. Haughian ${ }^{3}$, K. Hayama ${ }^{13}$, J.-F. Hayau ${ }^{46}$, J. Heefner ${ }^{1}$, A. Heidmann ${ }^{54}$, M. C. Heintze ${ }^{15}$, H. Heitmann ${ }^{45}$, P. Hello ${ }^{40}$, M. A. Hendry ${ }^{3}$, I. S. Heng ${ }^{3}$, A. W. Heptonstall ${ }^{1}$, V. Herrera ${ }^{30}$, M. Hewitson ${ }^{9}, 10$, S. Hild ${ }^{3}$, D. Hoak ${ }^{57}$, K. A. Hodge ${ }^{1}$, K. Holt ${ }^{8}$, M. Holtrop ${ }^{93}$, T. Hong ${ }^{64}$, S. Hooper ${ }^{43}$, D. J. Hosken ${ }^{94}$ J. Hough ${ }^{3}$, E. J. Howell ${ }^{43}$, B. Hughey ${ }^{14}$, S. Husa ${ }^{56}$, S. H. Huttner ${ }^{3}$, T. Huynh-Dinh ${ }^{8}$, D. R. Ingram ${ }^{19}$, R. Inta ${ }^{67}$, T. Isogai ${ }^{68}$, A. Ivanov ${ }^{1}$, K. Izumi ${ }^{13}$, M. Jacobson ${ }^{1}$, E. James ${ }^{1}$, Y. J. Jang ${ }^{83}$, P. Jaranowski ${ }^{34}$, E. Jesse ${ }^{90}$, W. W. Johnson ${ }^{61}$, D. I. Jones ${ }^{95}$, G. Jones ${ }^{70}$, R. Jones ${ }^{3}$, L. Ju ${ }^{43}$, P. Kalmus ${ }^{1}$, V. Kalogera ${ }^{83}$, S. Kandhasamy ${ }^{96}$, G. Kang ${ }^{97}$, J. B. Kanner ${ }^{55}$, R. Kasturi ${ }^{98}$,

E. Katsavounidis ${ }^{24}$, W. Katzman ${ }^{8}$, H. Kaufer ${ }^{9}, 10$, K. Kawabe ${ }^{19}$, S. Kawamura ${ }^{13}$, F. Kawazoe, ${ }^{9}$, , D. Kelley $^{23}$, W. Kells ${ }^{1}$, D. G. Keppel ${ }^{1}$, Z. Keresztes ${ }^{88}$, A. Khalaidovski $^{9}, 10$, F. Y. Khalili $^{39}$, E. A. Khazanov ${ }^{99}$, B. K Kim ${ }^{97}$, C. Kim ${ }^{100}$, H. Kim ${ }^{9,10}$, K. Kim ${ }^{101}$, N. Kim ${ }^{30}$, Y. M. Kim ${ }^{66}$, P. J. King ${ }^{1}$, D. L. Kinzel ${ }^{8}$, J. S. Kissel ${ }^{24}$, S. Klimenko ${ }^{15}$, K. Kokeyama ${ }^{16}$, V. Kondrashov ${ }^{1}$, S. Koranda ${ }^{14}$, W. Z. Korth ${ }^{1}$, I. Kowalska ${ }^{32}$, D. Kozak ${ }^{1}$, O. Kranz ${ }^{9,10}$, V. Kringel ${ }^{9,10}$, S. Krishnamurthy ${ }^{83}$, B. Krishnan ${ }^{20}$, A. Królak ${ }^{31,35}$, G. Kuehn ${ }^{9,10}$, R. Kumar ${ }^{3}$, P. Kwee ${ }^{10,9}$, P. K. Lam ${ }^{67}$, M. Landry ${ }^{19}$, B. Lantz ${ }^{30}$, N. Lastzka ${ }^{9,10}$, C. Lawrie ${ }^{3}$, A. Lazzarini ${ }^{1}$, P. Leaci ${ }^{20}$, C. H. Lee ${ }^{66}$, H. K. Lee ${ }^{101}$, H. M. Lee ${ }^{102}$, J. R. Leong ${ }^{9,10}$, I. Leonor ${ }^{53}$, N. Leroy ${ }^{40}$, N. Letendre ${ }^{4}$, J. Li ${ }^{59}$, T. G. F. Li ${ }^{11}$, N. Liguori77,78, P. E. Lindquist ${ }^{1}$, Y. Liu ${ }^{59}$, Z. Liu ${ }^{15}$, N. A. Lockerbie ${ }^{103}$, D. Lodhia ${ }^{16}$, M. Lorenzini ${ }^{51}$, V. Loriette ${ }^{41}$, M. Lormand ${ }^{8}$, G. Losurdo ${ }^{51}$, J. Lough ${ }^{23}$, J. Luan ${ }^{64}$, M. Lubinski ${ }^{19}$, H. Lück ${ }^{9,10}$, A. P. Lundgren ${ }^{44}$, E. Macdonald ${ }^{3}$, B. Machenschalk ${ }^{9,10}$, M. MacInnis ${ }^{24}$, D. M. Macleod ${ }^{70}$, M. Mageswaran ${ }^{1}$, K. Mailand ${ }^{1}$, E. Majorana $^{17}$, I. Maksimovic ${ }^{41}$,

N. Man ${ }^{45}$, I. Mandel ${ }^{24,16}$, V. Mandic ${ }^{96}$, M. Mantovani2 ${ }^{27,29}$, A. Marandi ${ }^{30}$, F. Marchesoni ${ }^{49}$, F. Marion ${ }^{4}$, S. Márka ${ }^{26}$, Z. Márka ${ }^{26}$, A. Markosyan $^{30}$, E. Maros ${ }^{1}$, J. Marque ${ }^{22}$, F. Martelli ${ }^{51,52}$, I. W. Martin ${ }^{3}$, R. M. Martin ${ }^{15}$, J. N. Marx ${ }^{1}$, K. Mason ${ }^{24}$, A. Masserot ${ }^{4}$, F. Matichard ${ }^{24}$, L. Matone ${ }^{26}$, R. A. Matzner ${ }^{85}$, N. Mavalvala ${ }^{24}$, G. Mazzolo ${ }^{9,10}$, R. McCarthy ${ }^{19}$, D. E. McClelland ${ }^{67}$, S. C. McGuire ${ }^{104}$, G. McIntyre ${ }^{1}$, J. McIver ${ }^{57}$, D. J. A. McKechan ${ }^{70}$, S. McWilliams ${ }^{26}$, G. D. Meadors ${ }^{60}$, M. Mehmet ${ }^{9,10}$, T. Meier ${ }^{10,9}$, A. Melatos ${ }^{69}$, A. C. Melissinos ${ }^{105}$, G. Mendell ${ }^{19}$, R. A. Mercer ${ }^{14}$, S. Meshkov ${ }^{1}$, C. Messenger ${ }^{70}$, M. S. Meyer ${ }^{8}$, H. Miao ${ }^{64}$, C. Michel ${ }^{47}$, L. Milano ${ }^{5,6}$, J. Miller ${ }^{67}$, Y. Minenkov ${ }^{71}$, V. P. Mitrofanov ${ }^{39}$, G. Mitselmakher ${ }^{15}$, R. Mittleman ${ }^{24}$, O. Miyakawa ${ }^{13}$, B. Moe ${ }^{14}$, M. Mohan ${ }^{22}$, S. D. Mohanty ${ }^{37}$, S. R. P. Mohapatra ${ }^{57}$, D. Moraru ${ }^{19}$, G. Moreno ${ }^{19}$, N. Morgado ${ }^{47}$, A. Morgia ${ }^{71,72}$, T. Mori ${ }^{13}$, S. R. Morriss ${ }^{37}$, S. Mosca ${ }^{5,6}$, K. Mossavi ${ }^{9,10}$, B. Mours ${ }^{4}$, C. M. Mow-Lowry ${ }^{67}$, C. L. Mueller ${ }^{15}$, G. Mueller ${ }^{15}$, S. Mukherjee ${ }^{37}$, A. Mullavey ${ }^{67}$, H. Müller-Ebhardt ${ }^{9,10}$, J. Munch ${ }^{94}$, D. Murphy ${ }^{26}$, P. G. Murray ${ }^{3}$, A. Mytidis ${ }^{15}$, T. Nash ${ }^{1}$, L. Naticchioni ${ }^{17,18}$, V. Necula ${ }^{15}$, J. Nelson ${ }^{3}$, I. Neri ${ }^{49,50}$, G. Newton ${ }^{3}$, T. Nguyen ${ }^{67}$, A. Nishizawa ${ }^{13}$, A. Nitz ${ }^{23}$, F. Nocera ${ }^{22}$,

D. Nolting ${ }^{8}$, M. E. Normandin ${ }^{37}$, L. Nuttall ${ }^{70}$, E. Ochsner ${ }^{14}$, J. O’Dell ${ }^{89}$, E. Oelker ${ }^{24}$, G. H. Ogin ${ }^{1}$, J. J. Oh ${ }^{91}$, S. H. Oh ${ }^{11}$, B. O'Reilly ${ }^{8}$, R. O'Shaughnessy ${ }^{14}$, C. Osthelder ${ }^{1}$, C. D. Ott ${ }^{64}$, D. J. Ottaway ${ }^{94}$, R. S. Ottens ${ }^{15}$, H. Overmier ${ }^{8}$, B. J. Owen ${ }^{44}$, A. Page ${ }^{16}$, G. Pagliaroli ${ }^{71,73}$, L. Palladino ${ }^{71,73}$, C. Palomba ${ }^{17}$, Y. Pan ${ }^{55}$, C. Pankow ${ }^{15}$, F. Paoletti ${ }^{27,22}$, M. A. Papa ${ }^{20,14}$, M. Parisi ${ }^{5,6}$, A. Pasqualetti ${ }^{22}$, R. Passaquieti ${ }^{27,28}$, D. Passuello ${ }^{27}$, P. Patel ${ }^{1}$, M. Pedraza ${ }^{1}$, P. Peiris ${ }^{86}$, L. Pekowsky ${ }^{23}$, S. Penn ${ }^{98}$, A. Perreca $^{23}$, G. Persichetti ${ }^{5,6}$, M. Phelps $^{1}$, M. Pichot ${ }^{45}$, M. Pickenpack ${ }^{9,10}$, F. Piergiovanni ${ }^{1,52}$, M. Pietka ${ }^{34}$, L. Pinard ${ }^{47}$, I. M. Pinto ${ }^{106}$, M. Pitkin ${ }^{3}$, H. J. Pletsch ${ }^{9,10}$, M. V. Plissi ${ }^{3}$, R. Poggiani27,28, J. Pöld ${ }^{9,10}$, F. Postiglione ${ }^{74}$, M. Prato ${ }^{65}$,

V. Predoi ${ }^{70}$, T. Prestegard ${ }^{96}$, L. R. Price ${ }^{1}$, M. Prijatelj ${ }^{9}, 10$, M. Principe ${ }^{106}$, S. Privitera ${ }^{1}$, R. Prix ${ }^{9,10}$, G. A. Prodii ${ }^{77,78}$, L. G. Prokhorov ${ }^{39}$, O. Puncken ${ }^{9,10}$, M. Punturo ${ }^{49}$, P. Puppo ${ }^{17}$,

V. Quetschke ${ }^{37}$, R. Quitzow-James ${ }^{53}$, F. J. Raab ${ }^{19}$, D. S. Rabeling ${ }^{11,12}$, I. Rácz ${ }^{76}$, H. Radkins ${ }^{19}$, P. Raffai ${ }^{87}$, M. Rakhmanov ${ }^{37}$, B. Rankins ${ }^{62}$, P. Rapagnani ${ }^{17,18}$, V. Raymond ${ }^{83}$, V. Re ${ }^{71,72}$, K. Redwine ${ }^{26}$, C. M. Reed ${ }^{19}$, T. Reed ${ }^{107}$, T. Regimbau ${ }^{45}$, S. Reid ${ }^{3}$, D. H. Reitze ${ }^{15}$, F. Ricci ${ }^{17,18}$, R. Riesen ${ }^{8}$, K. Riles ${ }^{60}$, N. A. Robertson ${ }^{1,3}$, F. Robinet ${ }^{40}$, C. Robinson ${ }^{70}$, E. L. Robinson ${ }^{20}$, A. Rocchi ${ }^{71}$, S. Roddy ${ }^{8}$, C. Rodriguez ${ }^{83}$, M. Rodruck ${ }^{19}$, L. Rolland ${ }^{4}$, J. G. Rollins ${ }^{1}$, J. D. Romano ${ }^{37}$, R. Romano ${ }^{5,7}$, J. H. Romie ${ }^{8}$, D. Rosińska ${ }^{33,36}$, C. Röver ${ }^{9}, 10$, S. Rowan ${ }^{3}$, A. Rüdiger ${ }^{9,10}$, P. Ruggi ${ }^{22}$, K. Ryan ${ }^{19}$, P. Sainathan ${ }^{15}$, F. Salemi ${ }^{9,10}$, L. Sammut ${ }^{69}$, V. Sandberg ${ }^{19}$, V. Sannibale ${ }^{1}$, L. Santamaría ${ }^{1}$, I. Santiago-Prieto ${ }^{3}$, G. Santostasi ${ }^{108}$, B. Sassolas ${ }^{47}$, B. S. Sathyaprakash ${ }^{70}$, S. Sato ${ }^{13}$, P. R. Saulson ${ }^{23}$, R. L. Savage ${ }^{19}$, R. Schilling ${ }^{9,10}$, R. Schnabel ${ }^{9,10}$, R. M. S. Schofield ${ }^{53}$, E. Schreiber ${ }^{9,10}$, B. Schulz ${ }^{9}, 10$, B. F. Schutz ${ }^{20,70}$, P. Schwinberg ${ }^{19}$, J. Scott ${ }^{3}$, S. M. Scott ${ }^{67}$, F. Seifert ${ }^{1}$, D. Sellers ${ }^{8}$, D. Sentenac ${ }^{22}$, A. Sergeev ${ }^{99}$, D. A. Shaddock ${ }^{67}$, M. Shaltev ${ }^{9,10}$, B. Shapiro ${ }^{24}$, P. Shawhan ${ }^{55}$, D. H. Shoemaker ${ }^{24}$, A. Sibley $^{8}$, X. Siemens ${ }^{14}$, D. Sigg ${ }^{19}$, A. Singer ${ }^{1}$, L. Singer ${ }^{1}$, A. M. Sintes ${ }^{56}$, G. R. Skelton ${ }^{14}$, B. J. J. Slagmolen ${ }^{67}$, J. Slutsky ${ }^{6}$, J. R. Smith ${ }^{2}$, M. R. Smith ${ }^{1}$, R. J. E. Smith ${ }^{16}$, N. D. Smith-Lefebvre ${ }^{24}$, K. Somiya ${ }^{64}$,

B. Sorazu ${ }^{3}$, J. Soto ${ }^{24}$, F. C. Speirits ${ }^{3}$, L. Sperandio ${ }^{71,72}$, M. Stefszky ${ }^{67}$, A. J. Stein ${ }^{24}$, L. C. Stein ${ }^{24}$, E. Steinert ${ }^{19}$, J. Steinlechner ${ }^{9,10}$, S. Steinlechner ${ }^{9,10}$, S. Steplewski ${ }^{48}$, A. Stochino ${ }^{1}$,

R. Stone ${ }^{37}$, K. A. Strain ${ }^{3}$, S. E. Strigin ${ }^{39}$, A. S. Stroeer ${ }^{37}$, R. Sturani ${ }^{51,52}$, A. L. Stuver ${ }^{8}$, T. Z. Summerscales ${ }^{109}$, M. Sung ${ }^{61}$, S. Susmithan ${ }^{43}$, P. J. Sutton ${ }^{70}$, B. Swinkels ${ }^{22}$, M. Tacca ${ }^{22}$,

L. Taffarello ${ }^{79}$, D. Talukder ${ }^{48}$, D. B. Tanner ${ }^{15}$, S. P. Tarabrin ${ }^{9,10}$, J. R. Taylor ${ }^{9,10}$, R. Taylor ${ }^{1}$, P. Thomas ${ }^{19}$, K. A. Thorne ${ }^{8}$, K. S. Thorne ${ }^{64}$, E. Thrane ${ }^{96}$, A. Thüring $^{10,9}$, K. V. Tokmakov ${ }^{103}$, C. Tomlinson ${ }^{75}$, A. Toncelli ${ }^{27,28}$, M. Tonelli ${ }^{27,28}$, O. Torre ${ }^{27,29}$, C. Torres ${ }^{8}$, C. I. Torrie ${ }^{1,3}$, E. Tournefier ${ }^{4}$, F. Travasso ${ }^{49,50}$, G. Traylor ${ }^{8}$, K. Tseng ${ }^{30}$, D. Ugolini $^{110}$, H. Vahlbruch ${ }^{10,9}$,

G. Vajente ${ }^{27,28}$, J. F. J. van den Brand ${ }^{11,12}$, C. Van Den Broeck ${ }^{11}$, S. van der Putten ${ }^{11}$, A. A. van Veggel ${ }^{3}$, S. Vass ${ }^{1}$, M. Vasuth ${ }^{76}$, R. Vaulin ${ }^{24}$, M. Vavoulidis ${ }^{40}$, A. Vecchio ${ }^{16}$, G. Vedovato ${ }^{79}$, J. Veitch ${ }^{70}$, P. J. Veitch ${ }^{94}$, C. Veltkamp ${ }^{9,10}$, D. Verkindt ${ }^{4}$, F. Vetrano ${ }^{51,52}$, A. Viceré ${ }^{51,52}$, A. E. Villar ${ }^{1}$, J.-Y. Vinet ${ }^{45}$, S. Vitale ${ }^{90,11}$, H. Vocca $^{49}$, C. Vorvick ${ }^{19}$, S. P. Vyatchanin $^{39}$, A. Wade $^{67}$, L. Wade ${ }^{14}$, M. Wade ${ }^{14}$, S. J. Waldman ${ }^{24}$, L. Wallace ${ }^{1}$, Y. Wan ${ }^{59}$, M. Wang ${ }^{16}$, X. Wang ${ }^{59}$, Z. Wang ${ }^{59}$, A. Wanner ${ }^{9}, 10$, R. L. Ward ${ }^{25}$, M. Was ${ }^{40}$, M. Weinert ${ }^{9}, 10$, A. J. Weinstein ${ }^{1}$, R. Weiss ${ }^{24}$, L. Wen ${ }^{64,43}$, P. Wessels ${ }^{9,10}$, M. West ${ }^{23}$, T. Westphal ${ }^{9,10}$, K. Wette $^{9,10}$, J. T. Whelan ${ }^{86}$, S. E. Whitcomb ${ }^{1,43}$, D. J. White ${ }^{75}$, B. F. Whiting ${ }^{15}$, C. Wilkinson ${ }^{19}$, P. A. Willems $^{1}$, L. Williams $^{15}$, R. Williams ${ }^{1}$, B. Willke ${ }^{9,10}$, L. Winkelmann ${ }^{9,10}$, W. Winkler ${ }^{9,10}$, C. C. Wipf ${ }^{24}$, A. G. Wiseman ${ }^{14}$, H. Wittel ${ }^{9,10}$, G. Woan ${ }^{3}$, R. Wooley ${ }^{8}$, J. Worden ${ }^{19}$, I. Yakushin ${ }^{8}$, H. Yamamoto ${ }^{1}$, K. Yamamoto ${ }^{9,10,78,80}$, C. C. Yancey ${ }^{55}$, H. Yang ${ }^{64}$, D. Yeaton-Massey ${ }^{1}$, S. Yoshida ${ }^{111}$, P. Yu ${ }^{14}$, M. Yvert ${ }^{4}$, A. Zadrożny ${ }^{35}$, M. Zanolin ${ }^{90}$, J.-P. Zendri ${ }^{79}$, F. Zhang ${ }^{59}$, L. Zhang ${ }^{1}$, W. Zhang ${ }^{59}$, C. Zhao ${ }^{43}$, N. Zotov ${ }^{107}$, M. E. Zucker ${ }^{24}$, and J. Zweizig ${ }^{1}$ 


\section{ABSTRACT}

Aims. The detection and measurement of gravitational-waves from coalescing neutron-star binary systems is an important science goal for groundbased gravitational-wave detectors. In addition to emitting gravitational-waves at frequencies that span the most sensitive bands of the LIGO and Virgo detectors, these sources are also amongst the most likely to produce an electromagnetic counterpart to the gravitational-wave emission. A joint detection of the gravitational-wave and electromagnetic signals would provide a powerful new probe for astronomy.

Methods. During the period between September 19 and October 20,2010, the first low-latency search for gravitational-waves from binary inspirals in LIGO and Virgo data was conducted. The resulting triggers were sent to electromagnetic observatories for followup. We describe the generation and processing of the low-latency gravitational-wave triggers. The results of the electromagnetic image analysis will be described elsewhere.

Results. Over the course of the science run, three gravitational-wave triggers passed all of the low-latency selection cuts. Of these, one was followed up by several of our observational partners. Analysis of the gravitational-wave data leads to an estimated false alarm rate of once every 6.4 days, falling far short of the requirement for a detection based solely on gravitational-wave data.

Key words. gravitational waves

\section{Introduction}

The direct detection and measurement of gravitational-waves from coalescing neutron-star and black-hole binaries is a highpriority science goal for ground-based gravitational-wave detectors. Event rate estimates suggest $\sim 1 / 100 \mathrm{y}$ detectable by the initial LIGO-Virgo network rising to $\sim 50 / y$ for the advanced LIGO-Virgo detector network (Abadie et al. 2010a). Coalescing compact binary systems containing at least one neutron star may also produce an electromagnetic counterpart to the gravitationalwave emission. Observation of both the gravitational and electromagnetic emission will allow astronomers to develop a complete picture of these energetic astronomical events.

Indeed there is a growing body of evidence that most short, hard $\gamma$-ray bursts (SHGRBs) are the result of binary neutron star or neutron star-black hole coalescence (Bloom et al. 2006; Berger et al. 2005; Villasenor et al. 2005; Berger 2009; Nakar et al. 2006). SHGRBs are known to emit electromagnetic radiation across the spectrum: From prompt, high energy emission that lasts $\sim$ minutes, to optical afterglows that decay on the order of hours and even radio emission that decays on the scale of weeks (Nakar \& Piran 2011). Coincident electromagnetic and gravitational observation of a SHGRB could confirm that the central engine of the $\gamma$-ray burst is indeed a coalescing compact binary.

If the $\gamma$-ray emission in SHGRBs is beamed, and the central engine is a compact binary merger, there will be many compact binary gravitational-wave events for which no $\gamma$-rays are observed by astronomers. On the other hand, optical afterglows may be observable further off-axis than the $\gamma$-rays albeit somewhat dimmer. There are other proposals for electromagnetic emission from binary neutron-star mergers including supernova-like emission due to the radioactive decay of heavy elements in the ejecta from the merger (Li \& Paczynski 1998; Metzger et al. 2010). This mechanism has been dubbed a "kilonova" since the luminosity peaks a thousand times higher than a typical nova. Kilonova emission is predicted to be isotropic and peaks roughly a day after merger. Current blind optical transient searches, such as that being undertaken by the Palomar Transient Factory, are only expected to find one of these events per year if the neutron binary coalescence rate is at the optimistic end of current estimates and that number increases substantially for the Large Synoptic Survey Telescope (LSST, Metzger et al. 2010). Since the network of gravitational-wave detectors is essentially omni-directional, there is a strong case to undertake an observing campaign in which compact-binary merger candidates, identified in gravitational-wave data, are rapidly followed up with electromagnetic observations.
In this paper we report on the first low-latency search for gravitational-waves from compact binary coalescence (CBC) in which detection candidates were followed up with optical observations. The search covered the period of time between September 19 and October 20, 2010, when LIGO detectors at Hanford, WA (H1), Livingston, LA (L1) and the Virgo detector in Cascina, Italy (V1) were jointly taking data. This time was contained within LIGO's sixth science run (S6) which began on July 7, 2009 and Virgo's third science run (VSR3) which began on August 11, 2010. The group of astronomical partners prepared to make followup observations included the Liverpool telescope, LOFAR, the Palomar Transient Factory, Pi of the Sky, QUEST, ROTSE III, SkyMapper, Swift, TAROT and the Zadko telescope. A companion paper (The LIGO Scientific Collaboration et al. 2012) describes more generally the process of collecting gravitational-wave triggers from a variety of transient searches, the human monitoring of the process and the production of telescope tilings.

The search resulted in three triggers that passed all of the selection cuts. The first occurred during a testing period and no alert was sent out. The second trigger was sent out to our astronomical partners and images were taken by several of them. An alert was issued for the third trigger, though its location was too close to the sun to be observed.

This paper is organized as follows: In Sect. 2 we provide a description of the analysis pipeline that produced triggers and localized them in the sky. In Sect. 3 we present a performance comparison between the low-latency pipeline used to generate astronomical alerts and the standard trigger generator used in offline searches for compact binary inspirals. Section 4 presents an overview of the run and the details of the triggers that were produced.

\section{The pipeline}

The major components of the analysis pipeline are shown in Fig. 1 and described in this section. Gravitational-wave data from the three participating detectors was first transfered to the Virgo site where it was processed by the Multi-Band Template Analysis (MBTA) to look for CBC signals. Interesting coincident triggers identified by MBTA were submitted to the Gravitational-wave Candidate Event Database (GraCEDb). A realtime alert system (LVAlert) was used to communicate information about these events to listening processes that automatically checked available information for possible anomalous detector behavior and estimated the location of the source on the sky. These automated steps completed within a few minutes after data acquisition. 


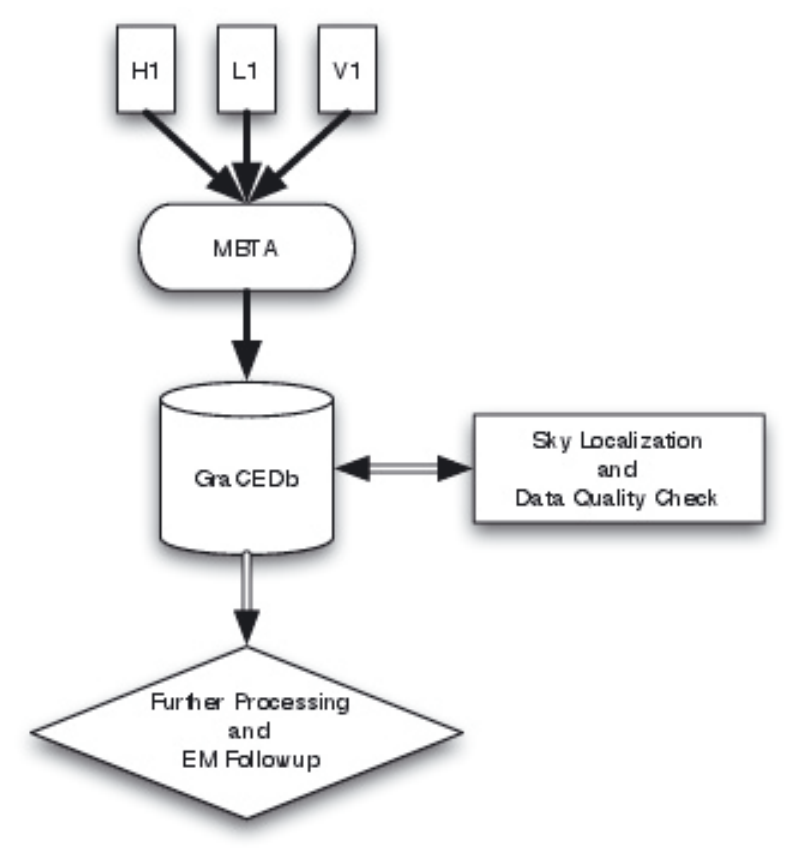

Fig. 1. An overview of the pipeline. Data produced at each of the three detector sites is transfered to a computer at Cascina where triggers are produced by MBTA and sent to GraCEDb for storage. Upon receiving events, GraCEDb alerts the sky localization and data quality check processes to begin and the results are then sent back to GraCEDb. If the triggers are localizable and of acceptable data quality then they are sent out for further processing and possibly followup by an optical telescope. The double stroke connections in the diagram are provided by LVAlert.

Further processing was needed before an alert was sent to our astronomical partners: human monitors reviewed information about the event, consulted the detector control rooms, and examined the data quality using a number of higher latency tools. Telescope image tilings were generated simultaneously in preparation for a positive decision to followup a trigger. The entire process took 20-40 min, with the largest latency incurred by the human monitor step. A histogram of the latency incurred before the trigger is sent out for further processing is shown in Fig. 2. Details about the rest of the event processing can be found in The LIGO Scientific Collaboration et al. (2012).

\subsection{Trigger production with MBTA}

\subsubsection{Pipeline structure and parameters}

The Multi-Band Template Analysis (MBTA) (Beauville et al. 2008) is a low-latency implementation of the standard matched filter (Wainstein \& Zubakov 1962) that is commonly used to search for gravitational-waves from compact binary coalescences (CBCs). As such, it relies on the accurate mathematical models of the expected signals to be used as search templates. Time domain templates with phase evolution accurate to second post-Newtonian order were used in the search. The search covered sources with component mass between 1-34 $M_{\odot}$ and total mass below $35 M_{\odot}$. A fixed bank of templates constructed with a minimal match of $97 \%$ was used to scan this parameter space. The template bank was constructed using a reference noise power spectrum taken at a time when the detectors were performing well. Given the detectors' typical noise spectra, the low-frequency cutoff of the templates was set to $50 \mathrm{~Hz}$, thus keeping the computational cost of the search light while losing negligible signal-to-noise ratio (SNR).

An original feature of MBTA is that it divides the matched filter across two frequency bands. This results in two immediate benefits: (1) The phase of the signal is tracked over fewer cycles meaning sparser template banks are needed in each frequency band and (2) a reduced sampling rate can be used for the lower frequency band, reducing the computational cost of the fast Fourier transforms involved in the filtering. The full band signal-to-noise ratio $\rho$ is computed by coherently combining the matched filtering outputs from the two frequency bands. The boundary between the low and high frequency bands is chosen such that the SNR is roughly equally shared between the two bands. The boundary frequencies range from $110 \mathrm{~Hz}$ to $156 \mathrm{~Hz}$, depending on the detector and on the mass ratio of the template bank of the individual MBTA processes.

A trigger is registered when $\rho>5.5$. Triggers are clustered across the template bank and across time: triggers less than $20 \mathrm{~ms}$ apart are recursively clustered under the loudest trigger. Clustered triggers are subjected to a $\chi^{2}$ test to check if the SNR distribution across the two frequency bands is consistent with the expected signal. The discriminating power of such a 2-band $\chi^{2}$ test is not as high as in typical implementations based on a larger number of frequency bands, but offers the advantage of having a negligible computational cost in the multi-band framework. The test can therefore be applied to all triggers at the single detector level. Triggers pass the $\chi^{2}$-test if

$\chi^{2}<3\left(2+0.025 \rho^{2}\right)$.

Single detector triggers that pass the $\chi^{2}$ test are tested for coincidence across detectors. Triggers from two detectors $i$ and $j$ are considered coincident if their time and mass parameters match within expected uncertainties. The mass coincidence criterion is

$\left|\mathcal{M}_{i}-\mathcal{M}_{j}\right|<\left(\frac{0.05}{M_{\odot}}\right)\left(\frac{\mathcal{M}_{i}+\mathcal{M}_{j}}{2}\right)^{2}$

where the chirp mass is given by $\mathcal{M}=\left(m_{1} m_{2}\right)^{3 / 5}\left(m_{1}+m_{2}\right)^{-1 / 5}$ in terms of the component masses, $m_{1}$ and $m_{2}$. The time coincidence criterion is

$\left|t_{i}-t_{j}\right|<\Delta t_{i j}$

where the maximum allowed time delays $\Delta t_{\mathrm{H} 1 \mathrm{~L} 1}=20 \mathrm{~ms}$ and $\Delta t_{\mathrm{H} 1 \mathrm{~V} 1}=\Delta t_{\mathrm{L} 1 \mathrm{~V} 1}=40 \mathrm{~ms}$ account for both the time of flight of the signal from one detector to another and for the experimental uncertainty in the arrival time measurement. The arrival time is measured by performing a quadratic fit around the maximum of the match filtered signal. It is then extrapolated to the time when the gravitational-wave signal is at a reference frequency chosen to minimize the statistical uncertainty on the measurement. This has the important effect of reducing the background by allowing tighter coincidence and improving the accuracy of position reconstruction by triangulation. It has been shown elsewhere (Acernese et al. 2007) that the optimal reference frequency is approximately that frequency where the detectors are most sensitive, although it depends somewhat on the mass of the source. A detailed study of simulated signals in S6/VSR3 noise resulted in the empirical parametrization $f_{\text {reference }}=\left[170-\mathcal{M}\left(5.1 / M_{\odot}\right)\right] \mathrm{Hz}$.

Triple detector coincidences are identified as a pair of H1L1 and $\mathrm{H} 1 \mathrm{~V} 1$ coincidences sharing the same $\mathrm{H} 1$ trigger. Although the pipeline identifies both double and triple coincidences, only the latter were submitted to GraCEDb, in line with the intention 
to focus on remarkable events, for which a sky location could be extracted by simple triangulation. Each triple has associated with it a combined SNR $\rho_{\mathrm{c}}$ defined by

$\rho_{c}^{2}=\sum_{j \in\{\mathrm{H} 1, \mathrm{~L} 1, \mathrm{~V} 1\}} \rho_{j}^{2}$

and a false alarm rate (FAR). Triggers with a smaller FAR, i.e. a larger $\rho_{\mathrm{c}}$, are more likely to be gravitational-waves. The nonstationary nature of the background means that a simple mapping from a given $\rho_{\mathrm{c}}$ to a corresponding FAR does not exist. Instead, the FAR must be explicitly calculated for each trigger.

The FAR is estimated as follows. Let $T_{i}$ be the analysis time needed to accumulate the last 100 triggers in detector $i$. Let $N_{\text {louder }}$ be the number of mass-coincidences that can be formed from the last 100 triggers in each detector for which $\rho_{c}$ is greater than the combined SNR of the observed coincidence. Then the expected rate of coincident triggers arising from background may be estimated as the product of the individual detector rates multiplied by a factor that accounts for the coincidence windows in time and mass. In particular, we define the FAR associated with a triple-coincident trigger to be

$\mathrm{F} A R=\alpha N_{\mathrm{louder}} \frac{4 \Delta t_{\mathrm{H} 1 \mathrm{~L} 1} \Delta t_{\mathrm{H} 1 \mathrm{~V} 1}}{T_{\mathrm{H} 1} T_{\mathrm{L} 1} T_{\mathrm{V} 1}}$

where $\alpha$ is an empirical factor with a value of 2 tuned to adjust the estimated FAR to the average observed rate of such triplecoincident triggers.

The low latency search is dependent on the short-order availability of the strain time series $h(t)$ measured by the detectors. Tools to reconstruct $h(t)$ with very low latency have been developed over the last few years, and had reached a mature state by the time of the S6/VSR3 runs. Calibration accuracies better than $15 \%$ on the amplitude of $h(t)$ were typically achieved for all detectors, which is quite sufficient for the purposes of our search.

For each detector, the $h(t)$ channel is produced at the experimental site computing center. The $\mathrm{H} 1$ and $\mathrm{L} 1 h(t)$ data are then transfered to the Virgo site computing center, where all the MBTA processing takes place. To minimize the latency, the communication of input/output data between the different processes involves no files on disk. It relies instead on the use of shared memories and of a TCP-IP based communication protocol developed for the Virgo data acquisition system. The whole set of processes ran on six computers.

\subsection{GraCEDb and LVAlert}

The storage and communication capabilities of the pipeline in Fig. 1 are provided by the GraCEDb and the LValert messaging system. The purpose of these technologies is to provide a continuously running system to ingest, archive and respond to gravitational-wave triggers. Communication with individual telescopes is handled at a later stage and uses whatever protocol is appropriate for the particular telescope.

The GraCEDb service stands behind an Apache $^{1}$ server and is built on Django ${ }^{2}$, a command line client that uses an HTTP/ReST (Fielding 2000; Fielding et al. 2002) interface to the server and authenticates with $\mathrm{X} 509^{3}$ certificates to a MySQL ${ }^{4}$

\footnotetext{
1 http://httpd.apache.org

2 https://www.djangoproject.com

${ }^{3}$ http://www.ietf.org/rfc/rfc2459.txt

${ }^{4}$ http://www . mysql.com
}

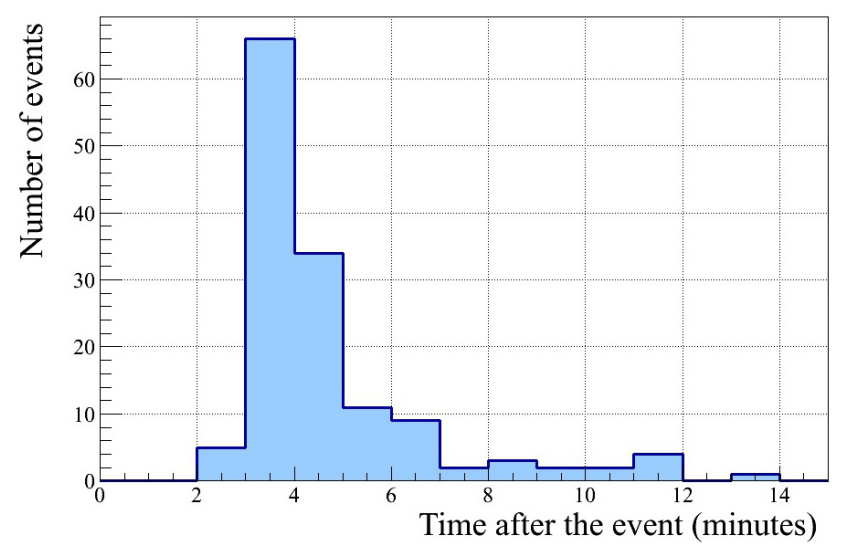

Fig. 2. Total latency of the automatic processes during the S6/VSR3 run. The $x$-axis is the difference between the time at which the trigger was available for human monitoring and the trigger's time. This includes an average of $63 \mathrm{~s}$ for all the data to be available at the Virgo site computing center and an average of $142 \mathrm{~s}$ for the trigger to be submitted to GraCEDb. This does not include the time for the human monitoring ( 20-40 $\mathrm{min}$ ) that takes place before an event is sent out for electromagnetic followup.

database back-end. A command-line client allows easy automation of event submission to GraCEDb. The prototype system used during S6/VSR3 was capable of ingesting triggers from MBTA and a number of other search pipelines. The raw trigger information was stored in an easily accessible archive and the most relevant information about the trigger, such as the time and significance, were ingested into the database. Upon successful ingestion, the trigger is given a unique identifier that is returned to the submitter. An alert is then sent out via LVAlert.

LVAlert is a communication client built on $\mathrm{XMPP}^{5}$ technology and the PubSub extension ${ }^{6}$. The system allows users to create nodes to which information can be published; users subscribe to nodes from which they want to receive that information. In the context of the current search, a node was set up for sending out alerts about MBTA triggers. A listener client is also provided that waits for alerts from the nodes to which the user is subscribed. By default, the listener simply prints any information it receives to the stdout, but it also allows users to develop plugins which can take action in response to the alerts. This is the mechanism for launching the data quality and sky localization jobs in Fig. 1.

\subsection{Event processing}

While MBTA is responsible for producing coincident triggers, there remains further processing to determine whether or not the triggers are suitable for external followups. In particular, this processing consists of performing sky localization, checking the quality of the data and determining whether or not the event is a known hardware injection, as described below. The mechanism for performing these tasks is an LVAlert listener that responds to alerts sent out by GraCEDb in response to new MBTA triggers.

The sky localization procedure proceeds by computing, as a function of location on the sky for a pre-determined grid, the offsets in timing and amplitude. Probabilities are assigned by comparing these measured quantities with distributions of these quantities obtained from simulated signals. More specifically,

\footnotetext{
5 http://xmpp.org

${ }^{6}$ http://xmpp.org/extensions/xep-0060.html
} 
the measure of timing accuracy used is

$\Delta t_{\mathrm{rss}}(\alpha, \delta)=\sqrt{\sum_{i \neq j}\left[\left(t_{i}(\alpha, \delta)-\hat{t}_{i}\right)-\left(t_{j}(\alpha, \delta)-\hat{t}_{j}\right)\right]^{2}}$,

where $t_{i}(\alpha, \delta)$ is the geocentric arrival time ${ }^{7}$ of a source located at right ascension $\alpha$ and declination $\delta$ in detector $i$ and $\hat{t}_{i}$ is the measured arrival time. To improve sky localization performance, the time of arrival is taken to be the time the signal crosses the reference frequency (Acernese et al. 2007) of $140 \mathrm{~Hz}$. The amplitude consistency parameter is given by

$\Delta Q_{\mathrm{rss}}(\alpha, \delta)=\sqrt{\sum_{i \neq j}\left(\frac{D_{\mathrm{eff}}^{(i) 2}-D_{\mathrm{eff}}^{(j) 2}}{D_{\mathrm{eff}}^{(i) 2}+D_{\mathrm{eff}}^{(j) 2}}-\frac{Q^{(i) 2}-Q^{(j) 2}}{Q^{(i) 2}+Q^{(j) 2}}\right)^{2}}$,

where the $(i)$ superscripts label the detector, $D_{\text {eff }}$ is the effective distance and we have suppressed the $(\alpha, \delta)$ of every quantity on the right hand side of the equation. Analytically, the effective distance is defined by

$D_{\text {eff }}=D\left[F_{+}^{2}\left(\frac{1+\cos ^{2} \iota}{2}\right)^{2}+F_{\times}^{2} \cos ^{2} \iota\right]^{-1 / 2}$,

where $D$ is the actual distance to the source, $F_{+, \times}=F_{+, \times}(\alpha, \delta, \psi)$ are the response functions of the detector (that depend on the polarization angle, $\psi)$ and $\iota$ is the inclination angle of the source, and the $(i)$ superscript is understood. In practice, the matched filtering procedure only provides a measurement $D_{\text {eff }}$ that is not typically invertible to obtain $D, F_{+, x}$, etc. We introduce the quantity (suppressing the $(i)$ superscript once again)

$$
Q^{2} \equiv \frac{1}{F_{+}^{2}(\theta, \phi, \psi=0)+F_{\times}^{2}(\theta, \phi, \psi=0)},
$$

to provide an ad hoc measure for determining the consistency of the amplitudes measured in each detector with a particular sky location. In practice, the use of this quantity improves the sky localization accuracy by roughly a factor of two, meaning it helps to break the mirror-image degeneracy inherent in a three-detector network when only triangulation is used to locate the source on the sky. Values of $\Delta t_{\mathrm{rss}}(\alpha, \delta)$ and $\Delta Q_{\mathrm{rss}}(\alpha, \delta)$ are assigned probabilities by comparing their values to those of a distribution of simulated signals. More specifically, simulated signals are placed into detector data and probability distributions are computed from the values of $\Delta t_{\text {rss }}\left(\alpha_{\text {true }}, \delta_{\text {true }}\right)$ and $\Delta Q_{\text {rss }}\left(\alpha_{\text {true }}, \delta_{\text {true }}\right)$, where the subscript indicates that the quantity is evaluated at the true location of the source in the sky. Since there is negligible correlation between the timing and amplitude measures, the normalized product of their values yields the probability distribution over the sky, i.e., the skymap.

As a demonstration of the performance of the sky localization routine, 10122 simulated signals were injected into data taken from the 6th week of S6, which overlapped with Virgo's second science run. The locations on the sky and distances were given by a galaxy catalog (Kopparapu et al. 2008). More details about these injections can be found in Sect. 3.2. The SNR distribution of the population of injections recovered by the pipeline is given in Fig. 3. We use two figures of merit to assess sky localization performance. The first is a quantity we call searched area. It is the area on the sky contained in the pixels that are assigned a probability greater than the probability assigned to the

\footnotetext{
7 We assume that gravitational-waves travel at the speed of light.
}

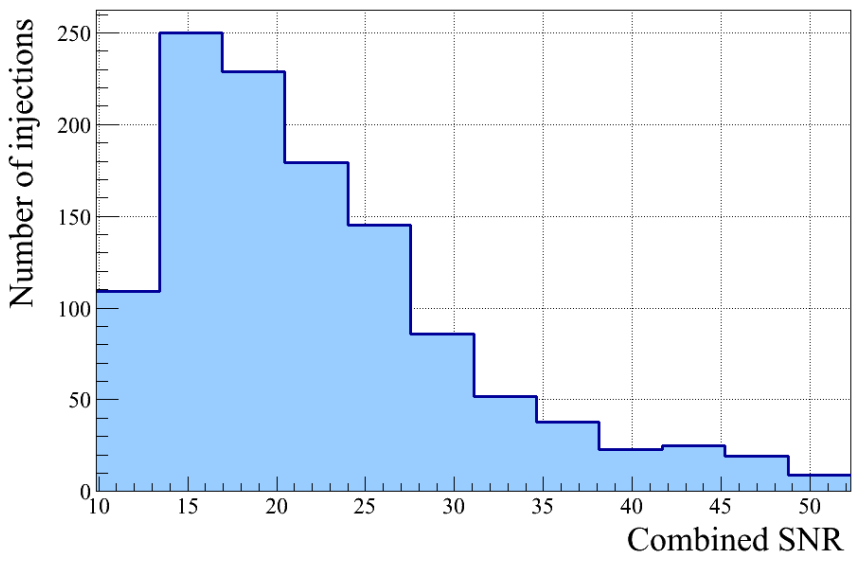

Fig. 3. The combined SNR distribution of the family of found injections used to demonstrate the sky localization performance. Emphasis was placed on weaker signals, where a first detection is more likely to arise.

pixel containing the true source location. In other words, it is the smallest area on the sky that one would be required to image to ensure the true source location is imaged. A complimentary figure of merit is the angular distance between the true source location and the most probable location identified by the sky localization routine. These figures of merit are shown as cumulative histograms in Fig. 4. To improve the performance of the sky localization routine, a galaxy catalog is used to guide the pointing in the following way: The skymap is weighted by the fraction of the cumulative blue luminosity (used in this context as a proxy for star formation rates) in each pixel out to the smallest effective distance measured by any of the detectors (The LIGO Scientific Collaboration et al. 2012). The effect of using a galaxy catalog is evident in the figures of merit shown in the upper curves in Fig. 4. Focusing on the lower curves, the angular distance figure of merit shows that for $60 \%$ of the injections the maximum likelihood point on the skymap is $\lesssim 5$ degrees away from the true source location, whereas the searched area is a much larger region on the sky. This indicates that although the maxima of the skymaps are close to the true source locations, they are quite broad. Application of the galaxy catalog does not do much to the angular distance histogram, but it eliminates large regions of the sky, effectively making the broad peaks much sharper. This can be seen explicitly in Fig. 13. In Fig. 5 the median searched area is plotted as a function of combined SNR. The upper panel depicts the results without the aid of the galaxy catalog, while the lower panel includes those improvements. A least squares fit of the median searched areas is given by the red lines. The fit is to a functional form $a_{0}+a_{-1} / \rho+a_{-2} / \rho^{2}$. The results that make use of the galaxy catalog should be understood as upper limits on the improvements a galaxy catalog can bring. As described in Sect. 3.2, the injection distribution was such that injections were more likely to come from galaxies with larger blue light luminosity. Although the choice to use the blue light luminosity is well motivated, it does not include, for example, binaries arising in elliptical galaxies, or the possibility that binaries follow some other property of their host galaxies. Better astrophysical priors are a subject we leave for future investigations.

Data quality plays an important role in offline searches, where detailed studies are performed to select data free from obvious instrumental or environmental artifacts by using information in auxiliary channels. While all the data quality information is not available with low latency, online production of a significant number of data quality flags was implemented during S6/VSR3. This allows for quick feedback on the current data 

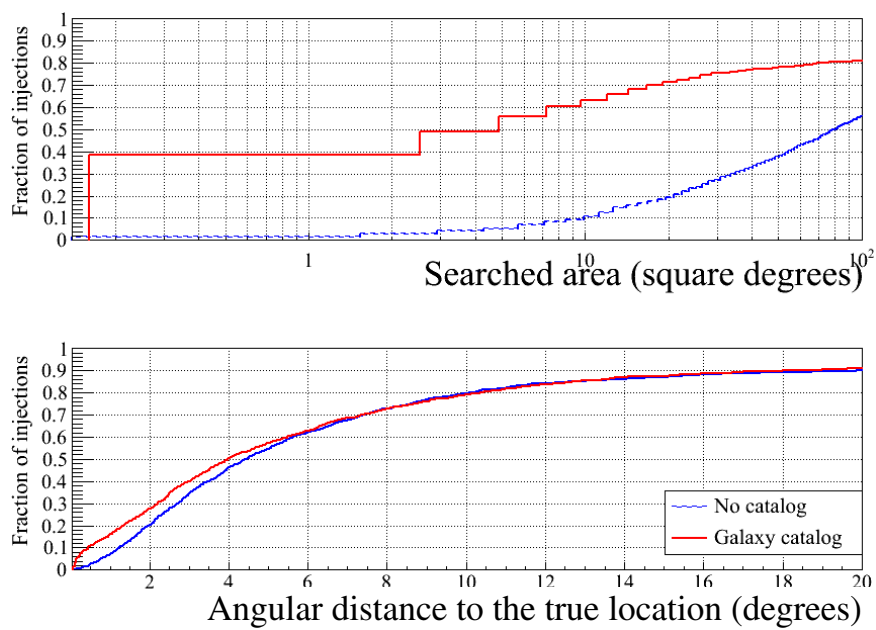

Fig. 4. Sky localization performance with and without the use of a galaxy catalog. The upper pane shows a cumulative histogram of the searched area in square degrees. The lower pane is a cumulative histogram of the angular distance, in degrees, between the injected location and the maximum likelihood recovered location. In both plots the red solid line is the performance with the aid of the galaxy catalog and the blue dotted line is the performance without the galaxy catalog.
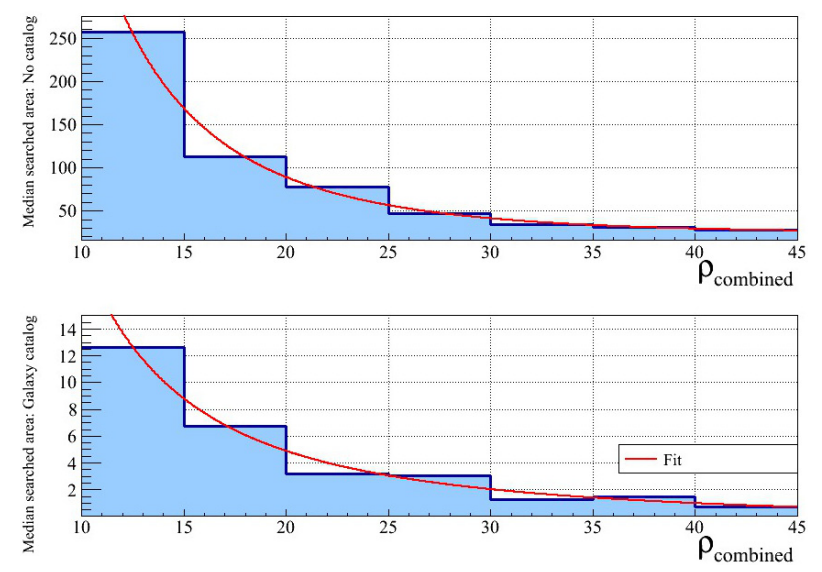

Fig. 5. Sky localization performance as a function of combined signalto-noise ratio with and without the use of a galaxy catalog (bottom and top panel, respectively). The blue bars indicate the median searched area, in square degrees, in each bin and the red lines depict a fit to these values.

quality, and to check the data at the time of a trigger against a list of trusted data quality flags produced online. As new data quality flags are developed and tested, the list is updated accordingly.

If an event passes all of our checks then it is sent out for further processing and possibly for electromagnetic observation.

\section{MBTA performance and validation}

In this section we look at the performance of MBTA. There are typically two ways in which performance is assessed: (1) through hardware injections, where the mirrors of each detector are physically displaced to simulate the presence of a signal and (2) software injections in which simulated signals are placed into detector data. We will first look at MBTA's performance on hardware injections and then provide a detailed comparison of its performance relative to the CBC offline pipeline (Abbott et al. 2008, 2007, 2009a,b; Abadie et al. 2010b), known as iHope, on a set of common software injections.

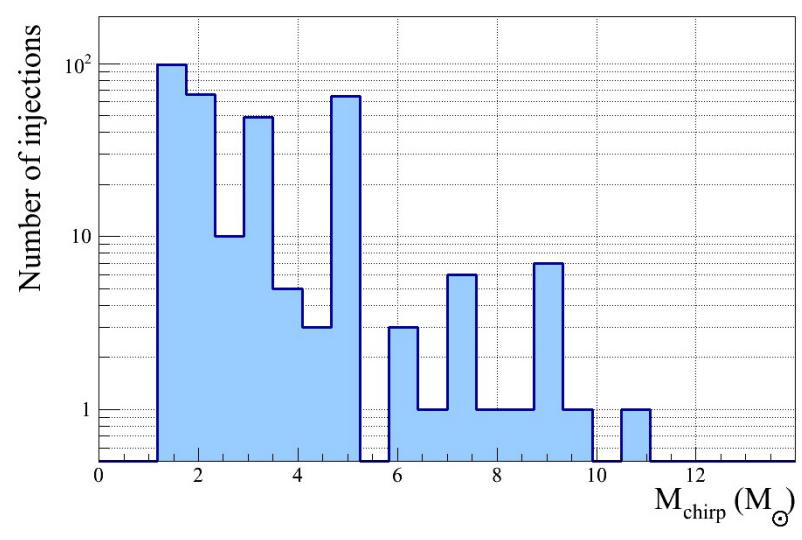

Fig. 6. The chirp mass distribution of all the hardware injections found in a single detector by both MBTA and the offline iHope pipeline during the S6/VSR3 run.

\subsection{Hardware injections}

Hardware injections are produced through the displacement of the mirror located at the end of arms. They are performed coherently in the three detectors, i.e. with injection time, amplitude and phase chosen in each detector to be consistent with the location of the source on the sky. During the S6/VSR3 scientific run, there were approximatively three hardware injections per day in each detector simulating the coalescence of a compact binary system, with a period of intensive injections between August 27, 2010 and September 3, 2010. For a number of reasons, not every injection was successful at every detector.

Two families of non-spinning waveforms were used for the hardware injections. The first family corresponds to analytic inspiral-merger-ringdown waveforms based on the Effective One-Body (EOB) model extended and tuned to match Numerical Relativity simulations of binary black hole coalescences (Buonanno et al. 2007; Pan et al. 2008). The second family of waveforms corresponds to restricted parameterized 2PN inspiral waveform computed in the time domain (Blanchet 1996; Arun et al. 2004). Among the hardware injections successfully performed in all three detectors, $62 \%$ are from the first family and $38 \%$ are from the second.

The parameters of the hardware injections were adjusted to appear in detector with an SNR $<100$. The total masses of the simulated binary systems were distributed between $2.8 M_{\odot}$ and $35 M_{\odot}$, with the masses of the components between $1.4 M_{\odot}$ and $35 M_{\odot}$. The hardware injections were distributed between $1 \mathrm{Mpc}$ and $80 \mathrm{Mpc}$, and the sky locations were chosen randomly. Figure 6 shows the chirp mass distribution of all the hardware injections which were successfully found by MBTA during the S6/VSR3 run.

An injection is considered to be recovered if MBTA produced a trigger with an end time within $20 \mathrm{~ms}$ of the end time of the injection. We restrict our attention to injections occurring when all three detectors are taking science quality data and all of the MBTA processes are up and running. Our focus is further narrowed by the requirement that the data quality is acceptable in 60 s preceding a trigger. This is to ensure that the Fourier transform required by the matched filter can be performed. Given these caveats, MBTA achieved a 93\% efficiency in detecting hardware injections in triple coincidence during the S6/VSR3 run. Figure 7 shows these recovered hardware injections and indicates the type of coincidence (double or triple) they were found in. 


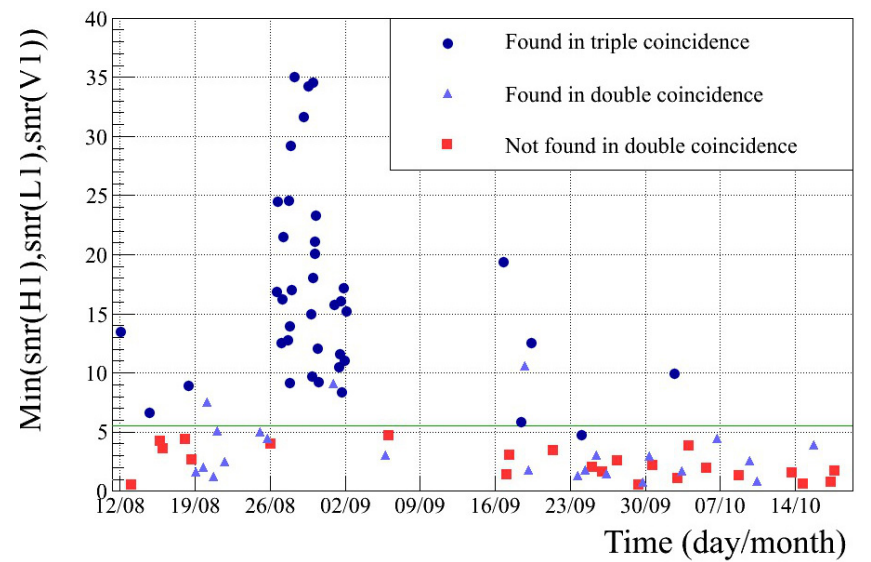

Fig. 7. This plot shows all the successful hardware injections which took place coherently in the three detectors. $93 \%$ of those which were above the threshold (expected SNR $\geq 5.5$ ) in each detector were detected in triple coincidence by MBTA.

An important step in the validation of MBTA is the comparison of the injected signal parameters with those recovered by MBTA. Figure 8 shows that the parameters of the injected signals are in good agreement with those recovered by MBTA. The first plot of Fig. 8 shows the timing accuracy parameter of the LIGO-Virgo network, $\Delta t_{\text {rss }}$, given by Eq. (6). The second and third plots of Fig. 8 show the difference between the chirp mass and effective distance of the binary injected and observed. From these three plots, it is clear that the low-latency search provides parameter estimations with comparable accuracy to that of the offline iHope pipeline.

\subsection{Software injections}

Hardware injections are for obvious reasons impractical for large scale performance studies. Instead, it is preferable to inject simulated signals into data that is already on disk. Here we will compare the performance of MBTA with the existing offline CBC pipeline, known as iHope. Both pipelines were run on a week of data containing simulated binary neutron star and neutron starblack hole binary signals. For this study a total of 10122 software injections were performed and analyzed in multiple parallel runs.

Simulated waveforms were generated using the stationary phase approximation (SPA) (Droz et al. 1999; Thorne 1987; Sathyaprakash \& Dhurandhar 1991) with the amplitude expanded to Newtonian order and the phase expanded to second post-Newtonian order with the upper cut-off frequency at the Schwarzschild innermost stable circular orbit (ISCO). Their locations were randomly chosen from the Compact Binary Coalescence Galaxy Catalogue (Kopparapu et al. 2008) in such a manner that the probability of choosing a galaxy is proportional to its blue light luminosity. Injections were made out to a distance of $40 \mathrm{Mpc}$ which roughly corresponds to the limits of sensitivity of the LIGO-Virgo network during the observation period, for the considered sources. The mass of each binary component ranged from $1 M_{\odot}$ to $15 M_{\odot}$ with the constraint on the total mass of the binary to be between $2 M_{\odot}$ and $20 M_{\odot}$. These choices were made to focus on systems likely to contain at least one neutron star, where an electromagnetic counterpart is expected.

After data quality vetoes were applied MBTA and iHope recovered different numbers of triple-coincident triggers,
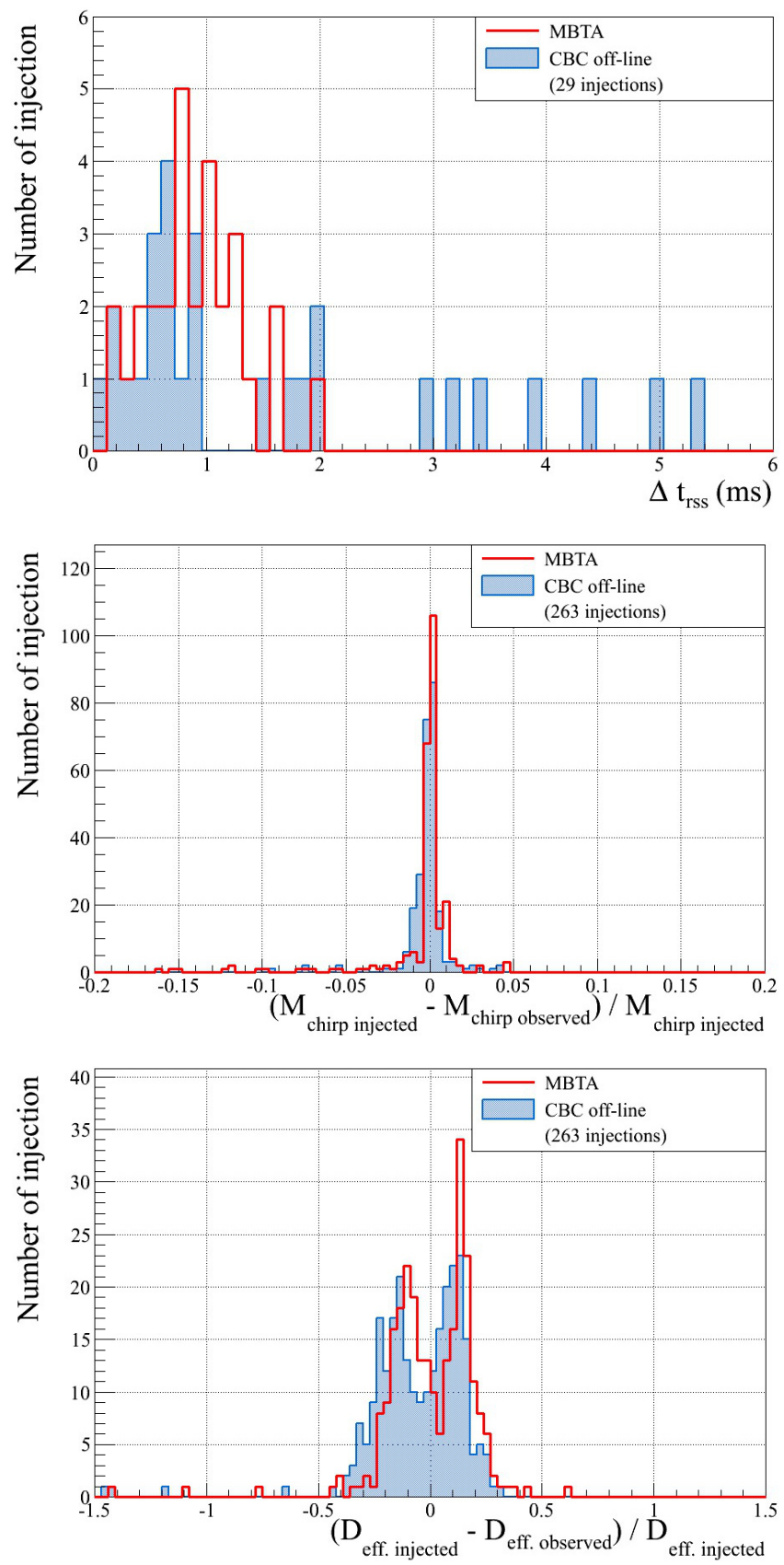

Fig. 8. These plots show the differences between the parameters of the hardware injections and those of the triggers found by both MBTA and the offline iHope pipeline. The first plot shows the timing accuracy parameter $\Delta t_{\mathrm{rss}}$ of all triple coincidences. The second plot corresponds to the difference between the chirp mass of the binary injected and observed. The third one corresponds to the difference between the effective distance of the binary injected and reconstructed by MBTA. The second and third plots contain all the hardware injections observed in $\mathrm{H} 1, \mathrm{~L} 1$ or V1.

736 and 859, respectively. Both pipelines recovered 709 identical injections in triple coincidence. Among the rest of the MBTA triples, twenty five were found by the offline CBC pipeline in double coincidence and the remaining two were completely missed. All these signals were near the threshold of detectability in one or more detectors.

Timing accuracy The primary goal of the low-latency pipeline is to send triggers out to the astronomical community for 


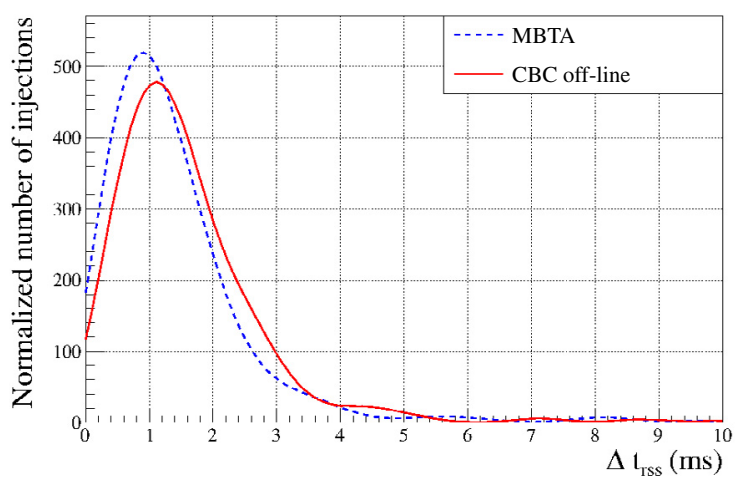

Fig. 9. Normalized distribution of timing accuracy of triggers detected by MBTA and CBC offline analysis pipeline. MBTA shows slightly better performance than the offline analysis.

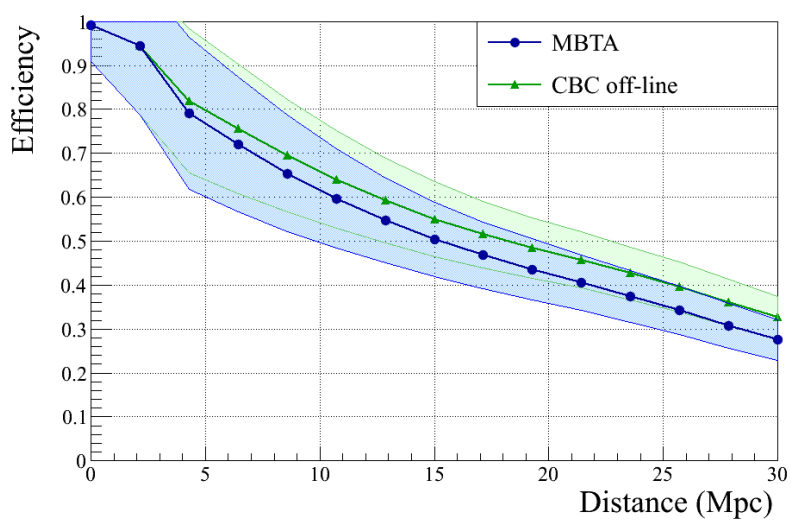

Fig. 10. Detection efficiency of MBTA and CBC offline analysis pipeline as a function of distance. Shaded regions indicate uncertainties. MBTA detects all simulated gravitational-wave signals injected in the nearby universe that the offline analysis does, but finds systematically less number of signals than the offline pipeline beyond $D>3 \mathrm{Mpc}$.

electromagnetic followup observations. Hence, localizing the GW candidate event on the sky is one of the essential parts of the search. Good timing accuracy for recovered injections is essential for good sky localization. Figure 9 shows the normalized distributions of the timing accuracy parameter of the LIGO-Virgo network, $\Delta t_{\mathrm{rss}}$, given by (6). From this plot it is clear that, overall, MBTA's performance in recovering the arrival time of gravitational-wave is better or comparable to that of the offline search. This is expected because MBTA uses a quadratic fit to find the peak of the SNR time series whereas the offline pipeline simply takes the maximum of the time series.

Efficiency as a function of distance is another key characteristic of an analysis pipeline. For each pipeline we measure its efficiency at recovering a GW signal as a triply coincident trigger, requiring also a false alarm rate less than or equal to 0.25 events per day, as for the alert generation. The result is shown in Fig. 10. At distances of about $3 \mathrm{Mpc}$, MBTA begins to systematically recover fewer signals than iHope. As expected this trend continues as both pipelines lose efficiency at large distances. Much of the decrease in efficiency of MBTA can be attributed to the fact that it imposes an effective threshold at a slightly higher SNR for a signal to be detected.

Chirp mass and SNR. Finally, Fig. 11 shows the SNRs and chirp masses in $\mathrm{H} 1$ recovered by the pipelines. They are in very
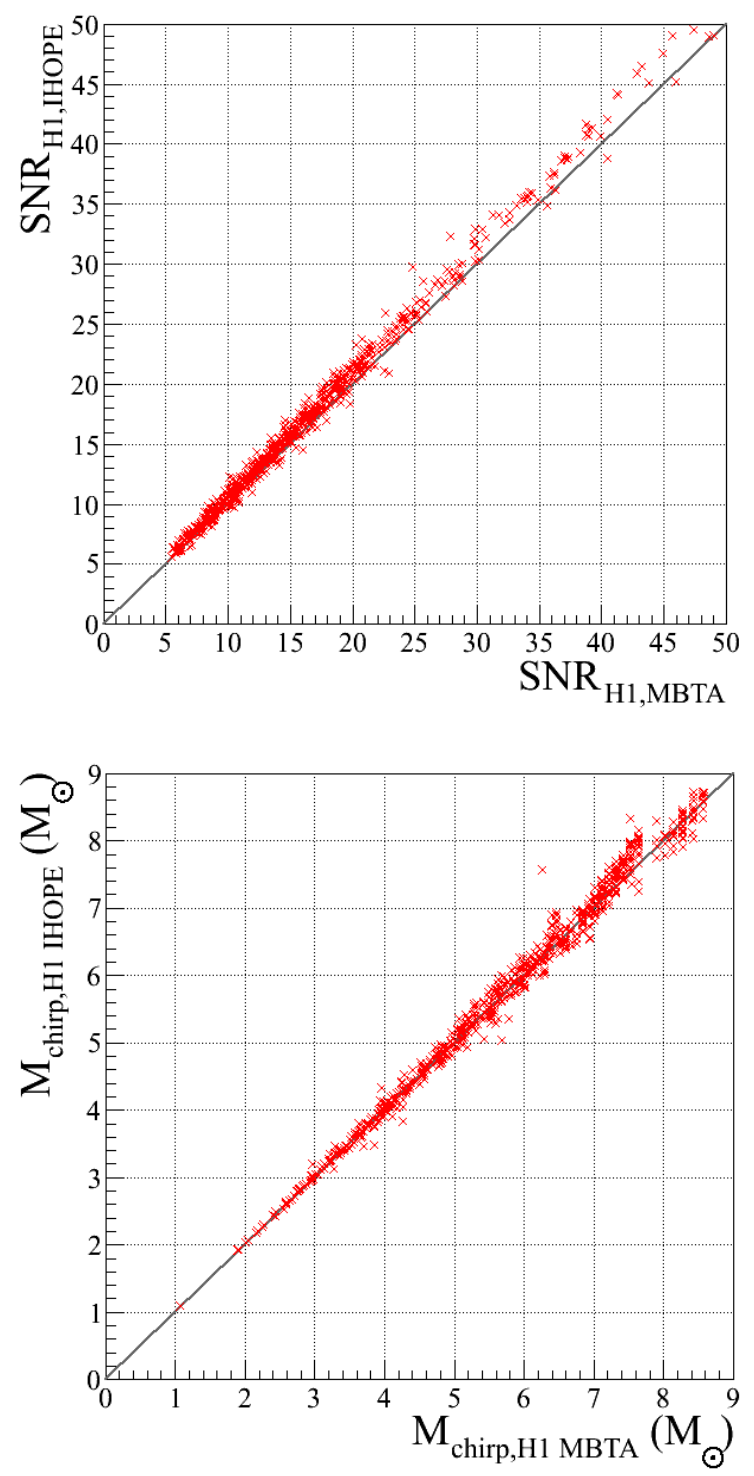

Fig. 11. Comparison of chirp mass and SNR between MBTA and CBC offline analysis pipeline.

good agreement with each other. The sparse density of the template bank at high mass shows up as some discreteness in the values of chirp mass recovered by MBTA, because MBTA uses a constant template bank, whereas iHope recomputes the noise power spectrum every $2048 \mathrm{~s}$ and a new template bank is produced in response. Similar agreement is found for the other two detectors.

In summary, the performance of MBTA is comparable to that of the offline pipeline.

\section{Results}

In this section we present the results of the analysis. We begin with an overview of the joint LIGO-Virgo science run before turning our attention to the triggers that passed the selection cuts.

\subsection{The S6/VSR3 run}

The joint LIGO/Virgo data taking started on August 11, 2010 when Virgo started its 3rd science run (VSR3), joining LIGO's 6th science run (S6). After a test and adjustment period during 
the first part of the S6/VSR3 run, the software of the low latency pipeline was frozen on August 27. From this time on, until the end of the run on October 19, the full pipeline was operating in production mode. The trigger production was first monitored to validate the pipeline during online operations and then the submission of EM alert was enabled on September 19.

During the production period of 52.6 days, the science mode duty cycle of the $\mathrm{H} 1, \mathrm{~L} 1$ and $\mathrm{V} 1$ detectors were respectively $63.9 \%, 64.8 \%$ and $69.7 \%$. The three detectors were operating simultaneously in science mode for a total time of 18.2 days, corresponding to a $34.6 \%$ duty cycle.

The duty cycle of MBTA during the triple coincidence time was $94.2 \%, 98.7 \%$ and $97.8 \%$ for the single H1, L1 and V1 triggers and $91.2 \%$ generating triggers in triple coincidence. Most of the down time occurred in one of a small number of periods lasting a few hours. The main problem was temporary network overloads between the LIGO Hanford site and Virgo Cascina site. The resulting delay in the arrival of $\mathrm{H} 1$ data prevented it from being used by MBTA.

Over the S6/VSR3 production period, 89 triple coincidences - including hardware injections - were detected by the MBTA pipeline and submitted to the GraCEDb database. A few of them (10) triggered multiple submissions of the same loud event corresponding to nearby "satellite" events. Three GraCEDb submissions failed. One because of a GraCEDb disk access problem. The other two failures were due to a problem with network authentication.

Future operations with improved configurations, software versions and monitoring tools are expected to reduce the down time of the pipeline which involved five different computing facilities located in Europe and North America.

\subsection{Triggers}

After removing the hardware injections, a total of 42 triple coincident triggers were observed during the search. The application of the online data quality flags reduced the number of triple coincident triggers from 42 to 37 . The time coincidence window was chosen conservatively (larger than the light travel time between sites) and, as a result, only 23 of these triggers were localizable on the sky.

At this stage of the pipeline, the triggers were sent out for further processing and alerts were generated for events having a false alarm rate of less than 0.25 events per day ( 1 event per day up to August 31). This cut reduced the number of triggers to 13 which were passed to the control rooms and on-call experts for further quality assessments.

Out of these 13 possible alerts generated, only 3 met the requirement of having at least one neutron $\operatorname{star}\left(M<3.5 M_{\odot}\right)$ associated with the merger. Table 1 gives a snapshot of the parameters of these three triggers.

The first trigger, G16901, from August 31, occurred during an initial testing period before alerts were sent out. A decision on this trigger was reached in the control room 14 min after the trigger time. The second trigger, G20190, on September 19, was accepted and images of the corresponding sky location were taken by Quest, ROTSE, SkyMapper, TAROT and Zadko. The decision to issue an alert was reached $39 \mathrm{~min}$ after the event occurred. The image analysis is in progress. Figure 12 shows the skymap produced for this trigger, and Fig. 13 shows the high probability region of the skymap, both with and without the galaxy catalog applied. The $90 \%$ confidence region was reduced from nearly 600 square degrees to 3.3 square degrees with the application of the galaxy catalog. The third trigger, G23201, on
Table 1. Parameters of the three triggers which passed all the selection cuts.

\begin{tabular}{|c|c|c|c|c|c|}
\hline Detecto & SNR & $\overline{\mathrm{eff}}[\mathrm{Mpc}$ & $\overline{m_{1}\left[M_{\odot}\right]}$ & $\overline{m_{2}\left[M_{\odot}\right]}$ & $\overline{\bar{M}\left[M_{\odot}\right]}$ \\
\hline \multicolumn{6}{|c|}{ G16901: $967254112 ;$ Combined SNR $=9.99 ; \mathrm{FAR}^{-1}=1.1$ days } \\
\hline H1 & 6.15 & 55 & 1.03 & 2.06 & 1.26 \\
\hline L1 & 5.61 & 54 & 1.36 & 1.38 & 1.19 \\
\hline V1 & 5.52 & 19 & 1.35 & 1.37 & 1.18 \\
\hline \multicolumn{6}{|c|}{ G20190: 968932960; Combined SNR $=10.0 ; \mathrm{FAR}^{-1}=6.4$ days } \\
\hline H1 & 6.07 & 99 & 2.94 & 3.00 & 2.59 \\
\hline L1 & 5.65 & 106 & 3.05 & 3.11 & 2.68 \\
\hline V1 & 5.60 & 27 & 2.23 & 4.15 & 2.62 \\
\hline \multicolumn{6}{|c|}{ G23201: 970399241; Combined SNR $=10.1 ; \mathrm{FAR}^{-1}=5.5$ days } \\
\hline $\mathrm{H} 1$ & 5.75 & 58 & 1.04 & 1.99 & 1.24 \\
\hline L1 & 5.84 & 41 & 0.98 & 1.95 & 1.19 \\
\hline V1 & 5.96 & 14 & 0.97 & 1.91 & 1.17 \\
\hline
\end{tabular}

Notes. See text for details.
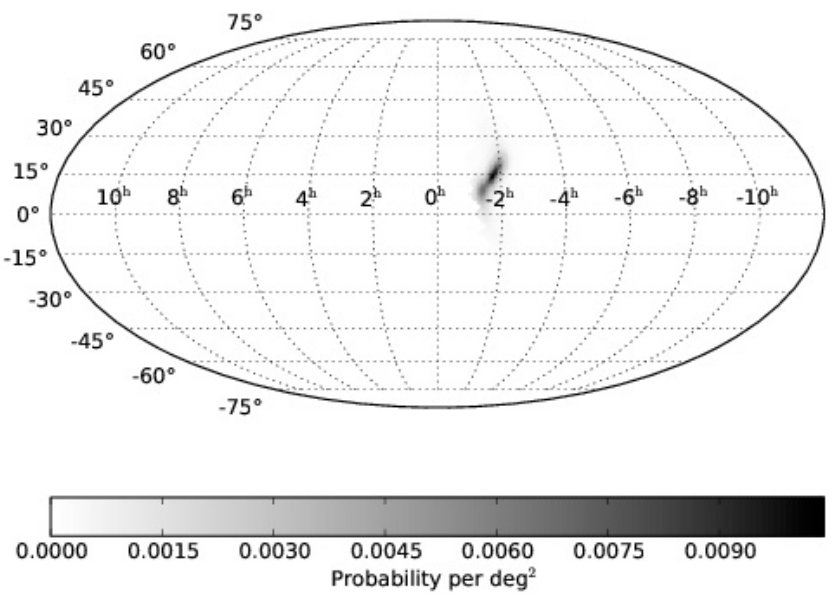

Fig. 12. Skymap for the G20190 trigger on September 19.

October 6, was unfortunately located too close to the sun, making it impossible to image. The decision to send the trigger out occurred $16 \mathrm{~min}$ after the trigger. Overall, these three triggers have SNR values close to the threshold value, with a false alarm rate of one per few days, typical of the expected background triggers. They are therefore not detection candidates on the basis of the gravitational-wave data analysis.

Figure 14 shows the cumulative rate of observed triggers as a function of the upper threshold applied on the estimated false alarm rate. The distribution focuses on triggers collected during the production period with a FAR less than 200 per year, requiring at least one neutron $\operatorname{star}\left(M<3.5 M_{\odot}\right)$ and excluding hardware injections. The vertical line indicates the threshold of FAR lower than $\sim 91$ per year (0.25 per day), which was used to determine which triggers were candidates for EM followup. This figure shows that the online FAR estimation is reasonable and therefore the background is under control. In particular, this figure shows no evidence of the FAR being underestimated, which is important since we do not want to unduly promote uninteresting triggers.

\section{Discussion}

The coalescence of binary systems containing neutron stars is the most promising source for the detection of both gravitational 

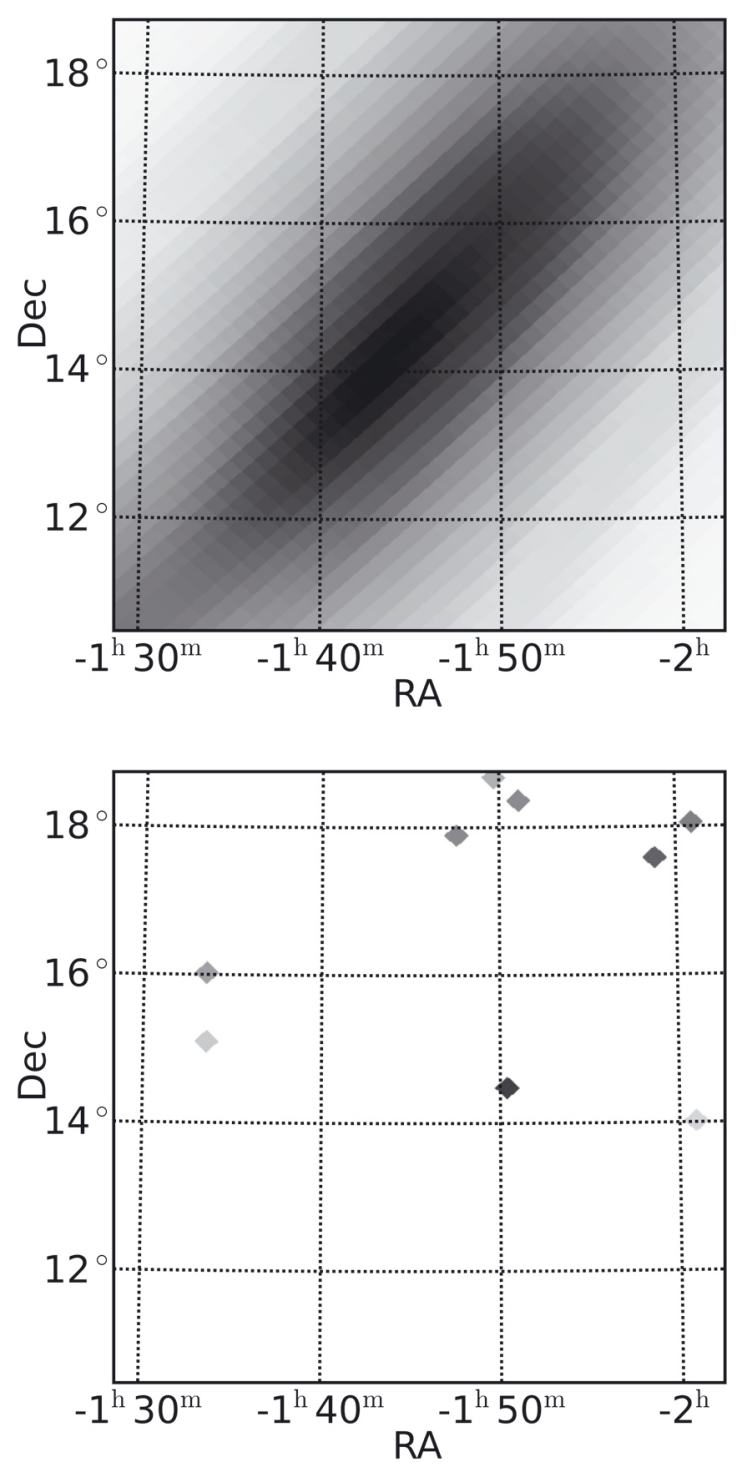

Fig. 13. Zoom into the high probability region of the skymap from G20190. The plot on top is generated solely from the gravitational-wave timing and amplitude offsets. The plot on the bottom is the result of applying the galaxy catalog as described in the text.

and electromagnetic radiation. We have presented the first lowlatency search for the gravitational-waves from these systems during the period between September 19 and October 20, 2010. The search resulted in a single trigger with a false alarm rate of one per 6.4 days being followed up with optical telescopes. The results of the image analysis are pending.

This exercise has resulted in a low-latency search for binary inspirals that performs on par with the standard offline pipeline. Triggers are produced on the scale of minutes and it is likely this can be further reduced. The limiting latency in sending triggers to electromagnetic observatories is the human monitoring involved. However, our demonstration that reliable false alarm rates can be computed rapidly suggests that this step could be removed in the advanced detector era.

Improvements of the pipeline will be explored in the future. For instance, the volume probed could be optimized by applying thresholds which could depend on the sensitivity and on the data quality of each detector. More detailed data quality information could help for this step. The search area in the sky might be reduced by using some coherent technique, possibly

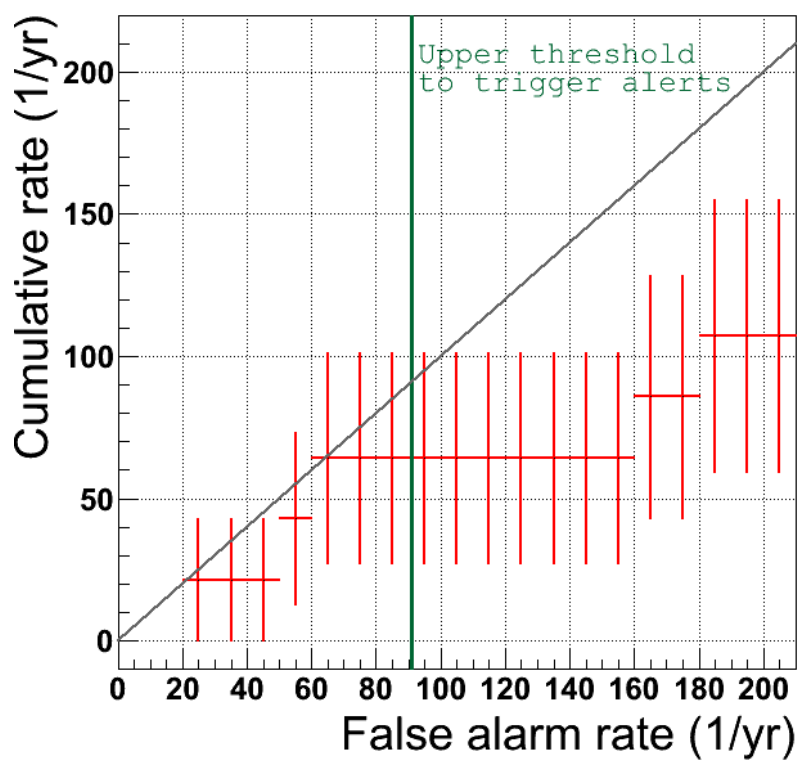

Fig. 14. Cumulative rate of triggers observed during the production period (excluding hardware injections) as a function of the upper threshold applied on the estimated false alarm rate.

in a hierarchical way. Extending the emission of fast alerts to significant double coincidences may also be useful.

Advanced LIGO and Virgo are likely to detect $~ 50$ neutron star coalescences per year (Abadie et al. 2010a). Successful observation of joint EM+GW emission depends crucially on using all the available information on these sources. Expectations for electromagnetic emission and their lightcurves, for example, will be important for designing optimal observing campaigns. In addition, even with a three-detector network, the sky localization ability is limited. More detectors, such as the LCGT or some other future detector will be important for increasing the chances of making successful joint EM+GW observations.

Acknowledgements. The authors gratefully acknowledge the support of the United States National Science Foundation for the construction and operation of the LIGO Laboratory, the Science and Technology Facilities Council of the United Kingdom, the Max-Planck-Society, and the State of Niedersachsen/Germany for support of the construction and operation of the GEO600 detector, and the Italian Istituto Nazionale di Fisica Nucleare and the French Centre National de la Recherche Scientifique for the construction and operation of the Virgo detector. The authors also gratefully acknowledge the support of gravitational-wave research by these agencies and by the Australian Research Council, the International Science Linkages program of the Commonwealth of Australia, the Council of Scientific and Industrial Research of India, the Istituto Nazionale di Fisica Nucleare of Italy, the Spanish Ministerio de Educación y Ciencia, the Conselleria d'Economia Hisenda i Innovació of the Govern de les Illes Balears, the Foundation for Fundamental Research on Matter supported by the Netherlands Organisation for Scientific Research, the Polish Ministry of Science and Higher Education, the FOCUS Programme of Foundation for Polish Science, the Royal Society, the Scottish Funding Council, the Scottish Universities Physics Alliance, The National Aeronautics and Space Administration, the Carnegie Trust, the Leverhulme Trust, the David and Lucile Packard Foundation, the Research Corporation, and the Alfred P. Sloan Foundation.

\section{References}

Abadie, J., Abbott, B. P., Abbott, R., et al. 2010a, Class. Quant. Grav., 27, 3001 Abadie, J., Abbott, B. P., Abbott, R., et al. 2010b, Phys. Rev. D, 81, 2001

Abbott, B., et al. (The LIGO Scientific Collaboration) 2007, Tuning matched filter searches for compact binary coalescence, Tech. Rep. LIGO-T070109-01 Abbott, B., Abbott, R., Adhikari, R., et al. 2008, Phys. Rev. D, 77, 062002 Abbott, B., Abbott, R., Adhikari, R., et al. 2009a, Phys. Rev. D, 79, 122001 Abbott, B., Abbott, R., Adhikari, R., et al. 2009b, Phys. Rev. D, 80, 047101 
Acernese, F., Amico, P., Alshourbagy, M., et al. 2007, Class. Quantum Grav., 24, S617

Arun, K. G., Blanchet, L., Iyer, B. R., \& Qusailah, M. S. S. 2004, Class. Quantum Grav., 21, 3771

Beauville, F., Bizouard, M.-A., Blackburn, L., et al. 2008, Class. Quantum Grav., 25, 045001

Berger, E. 2009, ApJ, 690, 231

Berger, E., Price, P. A., Cenko, S. B., et al. 2005, Nature, 438, 988

Blanchet, L. 1996, Phys. Rev. D, 54, 1417

Bloom, J. S., Prochaska, J. X., Pooley, D., et al. 2006, ApJ, 638, 354

Buonanno, A., Pan, Y., Baker, J. G., et al. 2007, Phys. Rev. D, 76, 104049

Droz, S., Knapp, D. J., Poisson, E., \& Owen, B. J. 1999, Phys. Rev. D, 59, 124016

Fielding, R. T. 2000, Ph.D. Thesis, University of California, Irvine

Fielding, R. T., Software, D., \& Taylor, R. N. 2002, ACM Transactions on Internet Technology, 2, 115

Kopparapu, R. K., Hanna, C., Kalogera, V., et al. 2008, ApJ, 675, 1459

Li, L.-X., \& Paczynski, B. 1998, Astrophys. J., 507, L59

Metzger, B. D. et al. 2010

Nakar, E., \& Piran, T. 2011

Nakar, E., Gal-Yam, A., \& Fox, D. B. 2006, ApJ, 650, 281

Pan, Y., Buonanno, A., Baker, J. G., et al. 2008, Phys. Rev. D, 77, 024014

Sathyaprakash, B. S., \& Dhurandhar, S. V. 1991, Phys. Rev. D, 44, 3819

The LIGO Scientific Collaboration, Virgo Collaboration: J. Abadie, Abbott, B. P., et al. 2012, A\&A, 539, A124

Thorne, K. S. 1987, in Three hundred years of gravitation, ed. S. W. Hawking, \& W. Israel (Cambridge: Cambridge University Press), 330

Villasenor, J. S., Lamb, D. Q., Ricker, G. R., et al. 2005, Nature, 437, 855

Wainstein, L. A., \& Zubakov, V. D. 1962, Extraction of signals from noise (Englewood Cliffs, NJ: Prentice-Hall)

1 LIGO - California Institute of Technology, Pasadena, CA 91125, USA

e-mail: larry.price@ligo.org

2 California State University Fullerton, Fullerton CA 92831 USA

3 SUPA, University of Glasgow, Glasgow, G12 8QQ, UK

4 Laboratoire d'Annecy-le-Vieux de Physique des Particules (LAPP), Université de Savoie, CNRS/IN2P3, 74941 Annecy-Le-Vieux, France

5 INFN, Sezione di Napoli, Complesso Universitario di Monte S.Angelo, 80126 Napoli, Italy

6 Università di Napoli "Federico II", Complesso Universitario di Monte S.Angelo, 80126 Napoli

7 Università di Salerno, Fisciano, 84084 Salerno, Italy

8 LIGO - Livingston Observatory, Livingston, LA 70754, USA

9 Albert-Einstein-Institut, Max-Planck-Institut Gravitationsphysik, 30167 Hannover, Germany

10 Leibniz Universität Hannover, 30167 Hannover, Germany

11 Nikhef, Science Park, Amsterdam, the Netherlands

12 VU University Amsterdam, De Boelelaan 1081, 1081 HV Amsterdam, the Netherlands

13 National Astronomical Observatory of Japan, Tokyo 181-8588, Japan

14 University of Wisconsin-Milwaukee, Milwaukee, WI 53201, USA

15 University of Florida, Gainesville, FL 32611, USA

16 University of Birmingham, Birmingham, B15 2TT, UK

17 INFN, Sezione di Roma, 00185 Roma, Italy

18 Università "La Sapienza", 00185 Roma, Italy

19 LIGO - Hanford Observatory, Richland, WA 99352, USA

20 Albert-Einstein-Institut, Max-Planck-Institut Gravitationsphysik, 14476 Golm, Germany

21 Montana State University, Bozeman, MT 59717, USA

22 European Gravitational Observatory (EGO), 56021 Cascina (PI), Italy

23 Syracuse University, Syracuse, NY 13244, USA

24 LIGO - Massachusetts Institute of Technology, Cambridge, MA 02139, USA

25 APC, AstroParticule et Cosmologie, Université Paris Diderot, CNRS/IN2P3, CEA/Irfu, Observatoire de Paris, Sorbonne Paris Cité, 10 rue Alice Domon et Léonie Duquet, 75205 Paris Cedex 13, France

26 Columbia University, New York, NY 10027, USA

27 INFN, Sezione di Pisa, 56127 Pisa, Italy
Università di Pisa, 56127 Pisa, Italy

29 Università di Siena, 53100 Siena, Italy

30 Stanford University, Stanford, CA 94305, USA

31 IM-PAN 00-956 Warsaw, Poland

32 Astronomical Observatory Warsaw University 00-478 Warsaw, Poland

33 CAMK-PAN 00-716 Warsaw, Poland

34 Białystok University 15-424 Białystok, Poland

35 NCBJ 05-400 Świerk-Otwock, Poland

36 Institute of Astronomy 65-265 Zielona Góra, Poland

37 The University of Texas at Brownsville and Texas Southmost College, Brownsville, TX 78520, USA

38 San Jose State University, San Jose, CA 95192, USA

39 Moscow State University, Moscow, 119992, Russia

40 LAL, Université Paris-Sud, IN2P3/CNRS, 91898 Orsay, France

41 ESPCI, CNRS, 75005 Paris, France

42 NASA/Goddard Space Flight Center, Greenbelt, MD 20771, USA

43 University of Western Australia, Crawley, WA 6009, Australia

44 The Pennsylvania State University, University Park, PA 16802, USA

45 Université Nice-Sophia-Antipolis, CNRS, Observatoire de la Côte d'Azur, 06304 Nice, France

46 Institut de Physique de Rennes, CNRS, Université de Rennes 1, 35042 Rennes, France

47 Laboratoire des Matériaux Avancés (LMA), IN2P3/CNRS, 69622 Villeurbanne, France

48 Washington State University, Pullman, WA 99164, USA

49 INFN, Sezione di Perugia, 06123 Perugia, Italy

50 Università di Perugia, 06123 Perugia, Italy

51 INFN, Sezione di Firenze, 50019 Sesto Fiorentino, Italy

52 Università degli Studi di Urbino "Carlo Bo", 61029 Urbino, Italy

53 University of Oregon, Eugene, OR 97403, USA

54 Laboratoire Kastler Brossel, ENS, CNRS, UPMC, Université Pierre et Marie Curie, 4 place Jussieu, 75005 Paris, France

55 University of Maryland, College Park, MD 20742 USA

56 Universitat de les Illes Balears, 07122 Palma de Mallorca, Spain

57 University of Massachusetts - Amherst, Amherst, MA 01003, USA

58 Canadian Institute for Theoretical Astrophysics, University of Toronto, Toronto, Ontario, M5S 3H8, Canada

59 Tsinghua University, Beijing 100084, China

60 University of Michigan, Ann Arbor, MI 48109, USA

61 Louisiana State University, Baton Rouge, LA 70803, USA

62 The University of Mississippi, University, MS 38677, USA

63 Charles Sturt University, Wagga Wagga, NSW 2678, Australia

64 Caltech-CaRT, Pasadena, CA 91125, USA

65 INFN, Sezione di Genova, 16146 Genova, Italy

66 Pusan National University, Busan 609-735, Korea

67 Australian National University, Canberra, ACT 0200, Australia

68 Carleton College, Northfield, MN 55057, USA

69 The University of Melbourne, Parkville, VIC 3010, Australia

70 Cardiff University, Cardiff, CF24 3AA, UK

71 INFN, Sezione di Roma Tor Vergata, 00133 Roma, Italy

72 Università di Roma Tor Vergata, 00133 Roma, Italy

73 Università dell' Aquila, 67100 L'Aquila, Italy

74 University of Salerno, 84084 Fisciano (Salerno), Italy and INFN (Sezione di Napoli), Italy

75 The University of Sheffield, Sheffield S10 2TN, UK

76 WIGNER RCP, RMKI, H-1121 Budapest, Konkoly Thege Miklós út 29-33, Hungary

77 INFN, Gruppo Collegato di Trento, 38050 Povo, Trento, Italy

78 Università di Trento, 38050 Povo, Trento, Italy

79 INFN, Sezione di Padova, 35131 Padova, Italy

80 Università di Padova, 35131 Padova, Italy

81 Inter-University Centre for Astronomy and Astrophysics, Pune 411007, India

82 California Institute of Technology, Pasadena, CA 91125, USA

83 Northwestern University, Evanston, IL 60208, USA

84 University of Cambridge, Cambridge, CB2 1TN, UK

85 The University of Texas at Austin, Austin, TX 78712, USA

86 Rochester Institute of Technology, Rochester, NY 14623, USA

87 Eötvös Loránd University, Budapest, 1117, Hungary 
88 University of Szeged, 6720 Szeged, Dóm tér 9, Hungary

89 Rutherford Appleton Laboratory, HSIC, Chilton, Didcot, Oxon OX11 0QX, UK

90 Embry-Riddle Aeronautical University, Prescott, AZ 86301, USA

91 National Institute for Mathematical Sciences, Daejeon 305-390, Korea

92 Perimeter Institute for Theoretical Physics, Ontario, N2L 2Y5, Canada

93 University of New Hampshire, Durham, NH 03824, USA

94 University of Adelaide, Adelaide, SA 5005, Australia

95 University of Southampton, Southampton, SO17 1BJ, UK

96 University of Minnesota, Minneapolis, MN 55455, USA

97 Korea Institute of Science and Technology Information, Daejeon 305-806, Korea

98 Hobart and William Smith Colleges, Geneva, NY 14456, USA
99 Institute of Applied Physics, Nizhny Novgorod, 603950, Russia

100 Lund Observatory, Box 43, 221 00, Lund, Sweden

101 Hanyang University, Seoul 133-791, Korea

102 Seoul National University, Seoul 151-742, Korea

103 University of Strathclyde, Glasgow, G1 1XQ, UK

104 Southern University and A\&M College, Baton Rouge, LA 70813 USA

105 University of Rochester, Rochester, NY 14627, USA

106 University of Sannio at Benevento, 82100 Benevento, Italy and INFN (Sezione di Napoli), Italy

107 Louisiana Tech University, Ruston, LA 71272, USA

108 McNeese State University, Lake Charles, LA 70609, USA

109 Andrews University, Berrien Springs, MI 49104, USA

110 Trinity University, San Antonio, TX 78212, USA

111 Southeastern Louisiana University, Hammond, LA 70402, USA 